\title{
Instability of a magnetoelastic layer resting on a non-magnetic substrate
}

\author{
K. Danas, ${ }^{\mathrm{a}, *}$, N. Triantafyllidis ${ }^{\mathrm{a}, \mathrm{b}}$ \\ ${ }^{a}$ Laboratoire de Mécanique des Solides, C.N.R.S. UMR7649 \& Département de Mécanique, \\ École Polytechnique, ParisTech, 91128 Palaiseau Cedex, France \\ ${ }^{b}$ Aerospace Engineering Department 83 Mechanical Engineering Department (emeritus) \\ The University of Michigan, Ann Arbor, MI 48109-2140 USA
}

\begin{abstract}
Magnetorheological elastomers (MREs) are ferromagnetic particle impregnated rubbers whose mechanical properties are altered by the application of external magnetic fields. Due to their coupled magneto-mechanical response, MREs are finding an increasing number of engineering applications. One such application is in haptics, where the goal is to actively control surface roughness. One way to achieve this is by exploiting the unstable regime of MRE substrate/layer assemblies subjected to transverse magnetic fields. In this work, we study the response of such an assembly subjected to a transverse magnetic field and in-plane stress. The layer is made up of a transversely isotropic MRE material, whose energy density has been obtained experimentally, while the substrate is a non-magnetic isotropic pure polymer/gel. An analytical solution to this problem based on a general, finite strain, 2D continuum modeling for both the MRE layer and the substrate, shows that for adequately soft substrates there is a finite-wavelength buckling mode under a transverse magnetic field. Moreover, the critical magnetic field can be substantially reduced in the presence of a compressive stress of the assembly, thus opening the possibility for haptic applications operating under low magnetic fields.
\end{abstract}

Key words: Magnetorheological elastomers, Thin films, Finite strain, Substrate, Nonlinear elasticity, Instabilities

\footnotetext{
${ }^{*}$ Corresponding author.

Email addresses: kdanas@lms.polytechnique.fr (K. Danas,), nick@lms.polytechnique.fr (N. Triantafyllidis) 


\section{Contents}

1 Introduction $\quad 3$

2 Problem formulation $\quad 4$

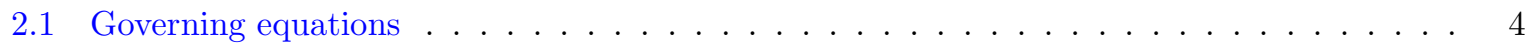

2.2 Geometry and loading . . . . . . . . . . . . . . . . . . . . . . 5

2.3 Material selection . . . . . . . . . . . . . . . . . . . . . . . . . 7

2.4 Principal solution . . . . . . . . . . . . . . . . . . . . . 8

3 Onset of bifurcation $\quad \mathbf{8}$

3.1 Euler-Lagrange equations for the MRE layer . . . . . . . . . . . . . . . . . . . . . . . . . . . . .

3.2 Euler-Lagrange equations for the substrate . . . . . . . . . . . . . . . . . 10

3.3 Euler-Lagrange equations for the air . . . . . . . . . . . . . . . . . . . 11

3.4 Determination of critical loads and corresponding eigenmodes . . . . . . . . . . . . 11

4 Results and discussion $r$

4.1 Bifurcation due to purely magnetic loading . . . . . . . . . . . . . . . . . . 12

4.2 Bifurcation due to purely mechanical loading . . . . . . . . . . . . . . . . . . 14

4.3 Bifurcation due to combined magnetic and mechanical loading . . . . . . . . . . . . . . 15

$\begin{array}{llr}5 & \text { Conclusion } & 17\end{array}$

$\begin{array}{lr}\text { A Bifurcation equations coefficients } & \mathbf{2 0}\end{array}$

A.1 MRE layer . . . . . . . . . . . . . . . . . . . . . . . . . 20

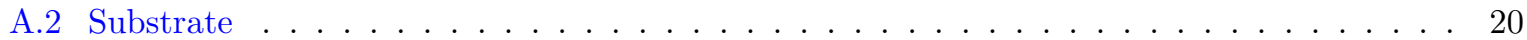

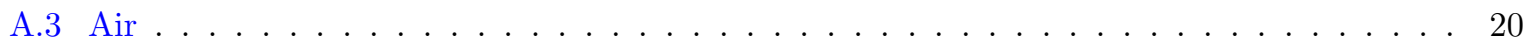

B First and second derivatives of the energy density functions 21

$\begin{array}{lr}\text { C Coefficients of the matrix } \mathcal{D} & 21\end{array}$

D Asymptotic result for compliant substrates $r$ 


\section{Introduction}

Magnetorheological elastomers (MREs) are ferromagnetic particle impregnated rubbers that can be deformed by external magnetic fields (e.g. see Rigbi and Jilkén (1983); Ginder et al. (1999)). These composites are members of a wide class of materials that exhibit multi-physics couplings and termed "active materials". In virtue of the strong interactions between their magnetic and elastic response, MRE's are considered for a wide number of engineering applications, such as sensors, actuators, vibrations dampers or haptic devices. It is this latter category of applications that motivates the present study and more specifically the possibility of inducing deformation patterns in MRE layers through external magnetic fields and controlled by mechanical loads.

More specifically, of interest here is a MRE layer bonded on a non-magnetic substrate and subjected to a combination of magnetic and mechanical loads: transverse (along the thickness direction) magnetic field and axial (parallel to the interface) compression. Either loading by itself can lead to bifurcation (buckling) of the MRE layer. For the purely magnetic loading case, Moon and Pao (1968) studied the problem of magnetoelastic buckling of a thin metallic ferromagnetic plate subjected to a transverse magnetic field using structural models. In that work, the plate is initially flat and stable. When the magnetic field reaches a critical value, the plate rotates and the corresponding buckling wavelength is comparable to the finite length of the thin plate. The instability of the plate subjected to a transversely magnetic field can be interpreted as the well-known compass effect in magnetism, where an unconstrained rod/plate tends to align with the applied magnetic field. In the present work, the presence of the non-magnetic substrate penalizes the energy of the long wavelength modes in the unbounded MRE layer/substrate system, leading to finite wavelengths for the critical instability mode (i.e., mode corresponding to the lowest value of the applied external magnetic field).

In a subsequent study, Pao and Yeh (1973) revisited this problem using a continuum theory of magnetoelasticity which, upon linearization, yields the buckling results of Moon and Pao (1968). In addition to these theoretical investigations, one should also mention associated experimental studies (e.g. Wallerstein and Peach (1972), Popelar (1972), Miya et al. (1978)). The bifurcation problem of an MRE block under plane strain conditions and subjected to a transverse magnetic field has recently been investigated by Kankanala and Triantafyllidis (2008) using the coupled magneto-mechanical variational formulation proposed by Kankanala and Triantafyllidis (2004). The structural model results of Moon and Pao (1968) have been recovered from the 2D analysis of Kankanala and Triantafyllidis (2008) in the asymptotic limit of a vanishing block aspect ratio.

The problem of purely mechanical loading for the substrate/layer assembly has attracted considerably more attention. There, the instability is due to the axial compression of the subspace, a phenomenon initially pointed out by Biot (1965). The addition of a stiffer thin film bonded on a softer substrate has been the object of numerous subsequent studies (the interested reader is referred to Shield et al. (1994), Chen and Hutchinson (2004), Huang et al. (2005), Audoly and Boudaoud (2008a) and references quoted therein). The combination of mechanical and magnetic loading to study the stability of composites has appeared very recently with the work of Rudykh and Bertoldi (2013), who have investigated infinite, magnetoactive layered composites. In the present work, and keeping in mind our interest in haptic applications, the goal is to choose the constitutive properties of the MRE layer and the substrate in combination with appropriately applied magnetic and mechanical loading conditions in order to achieve buckling at rather low magnetic fields.

The model studied here consists of an infinite MRE layer perfectly bonded to a non-magnetic substrate and subjected to a transverse magnetic field and lateral in-plane compression. The layer is made up of a transversely isotropic MRE, while the substrate is a non-magnetic isotropic elastomer. A variational, energy-based formulation for conservative problems is used and an analytical solution of the associated 2D (plane strain) problem is presented. More specifically, in Section 2, we present the governing equations of the problem, based on the general variational framework of Kankanala and Triantafyllidis (2004). In this same section, we define the geometry of the problem, select the material properties following the experimental results of Danas et al. (2012) and present the principal solution of the MRE substrate/layer assembly. In Section 3, we describe the bifurcation analysis of the assembly for arbitrary constitutive laws. In Section 4, we present results of the critical applied magnetic field $\widehat{h}_{c}$ and critical stretch ratio of the interface $\lambda_{1}^{c}$ (a measure of the applied compressive stress) as a function of the MRE substrate/layer shear moduli ratio $G_{s} / G_{l}$. In the case of purely magnetic loading $\left(\lambda_{1}=1\right)$, because of magnetic saturation the critical magnetic field is found to increase rapidly with $G_{s} / G_{l}$ leading to $\widehat{h}_{c}$ values that are unrealistic for applications. For the same reason, a bifurcation due to a purely magnetic loading becomes 
impossible except for very soft substrates. By contrast, a combination of mechanical (compressing the MRE substrate/layer assembly with $\lambda_{1}<1$ near its purely mechanical instability threshold) and magnetic loads, brings down substantially the critical magnetic field and the assembly can bifurcate even for substrates that are stiffer than the layer. The MRE layer anisotropy is found to play an important role in the selection of the critical wavelength. Concluding remarks and suggestions for future work are presented in Section 5. For efficiency, the lengthy intermediate steps in the analysis are given in Appendix, whose last section provides a simple, physically motivated structural model that helps the reader understand why a thin, stiff layer bonded on a soft substrate buckles under a transverse magnetic field and establishes key asymptotic results.

\section{Problem formulation}

This section presents the equations governing the layer-substrate system under magneto-mechanical loading conditions. More specifically, we first present the variational formulation of the problem and the resulting governing equations (the point-wise Euler-Lagrange equations plus the boundary/interface conditions). We then define the layer-substrate geometry and describe the applied loading. Subsequently, we present the energy density function for the MRE-layer and the non-magnetic substrate. The section concludes with the derivation of the principal solution.

\subsection{Governing equations}

Following the work of Kankanala and Triantafyllidis (2004, 2008), we assume that the potential energy $\mathcal{P}$ of the system under consideration can be written as (see Eq.(3.1) in (Kankanala and Triantafyllidis, 2004) for a detailed proof)

$$
\mathcal{P}=\int_{V} \rho_{0}[\psi(\mathbf{F}, \mathbf{M}, \mathbf{N})-\mathbf{f} \cdot \mathbf{u}] \mathrm{d} V-\int_{\partial V} \mathbf{T} \cdot \mathbf{u} \mathrm{d} S+\int_{\mathbb{R}^{3}} \frac{\mu_{0} J}{2} \mathbf{h} \cdot \mathbf{h} \mathrm{d} V .
$$

In this expression, $\psi$ denotes the specific Helmholtz free energy function which, for both layers of interest depends upon the deformation gradient $\mathbf{F} \equiv \mathbf{x} \nabla^{1}$, and for the case of the MRE-layer depends also on the current specific magnetization $\mathbf{M}$ and on a vector $\mathbf{N}$ used to describe its anisotropy. The reference density $\rho_{0}$ is related to the current density $\rho$ by $\rho_{0}=\rho J$ (with $J \equiv \operatorname{det} \mathbf{F}$ ), $\mathbf{T}$ is the mechanical surface traction vector in the reference configuration and $\mathbf{f}$ is the mechanical specific body force vector. Notice that the magnetic energy in (2.1) is integrated not over the volume $V$ occupied by the solid but over the entire space $\mathbb{R}^{3}$, since the magnetic field extends in the surrounding air; the constant appearing in front of the magnetic energy $\mu_{0}=4 \pi 10^{-7} N A^{-2}$ is the magnetic permeability of vacuum.

The magnetic loading consists of applying an external h-field $\widehat{\mathbf{h}}$. The total current h-field $\mathbf{h}$ (or the total current magnetic field $\mathbf{b}$ ) are decomposed into the externally applied h-field $\widehat{\mathbf{h}}$ (or externally applied magnetic field $\widehat{\mathbf{b}}$ ) plus the corresponding perturbation fields $\widetilde{\mathbf{h}}$ (or $\widetilde{\mathbf{b}}$ ) as follows:

$$
\mathbf{h}=\widehat{\mathbf{h}}+\widetilde{\mathbf{h}}, \quad \mathbf{b}=\widehat{\mathbf{b}}+\widetilde{\mathbf{b}}, \quad \widehat{\mathbf{b}}=\mu_{0} \widehat{\mathbf{h}}, \quad \widetilde{\mathbf{b}}=\mu_{0}(\widetilde{\mathbf{h}}+\rho \mathbf{M}) .
$$

Moreover, using the fact that the current magnetic field is divergence free $(\boldsymbol{\nabla} \cdot \mathbf{b}=0)$, one can show that the perturbed magnetic field $\widetilde{\mathbf{B}}$ in the reference configuration takes the form:

$$
\widetilde{\mathbf{B}}=J \mathbf{F}^{-1} \cdot \widetilde{\mathbf{b}}, \quad \widetilde{\mathbf{B}}=\nabla \times \widetilde{\mathbf{A}},
$$

where $\widetilde{\mathbf{A}}$ is the vector potential of the magnetic field perturbation in the reference configuration.

Substituting the above equations in the original potential energy (2.1), we rewrite $\mathcal{P}$ as a function of three independent variables: the displacement $\mathbf{u}$, the current specific magnetization $\mathbf{M}$ and the vector potential $\widetilde{\mathbf{A}}$ of the magnetic field perturbation, resulting (see Eq.(3.12) (Kankanala and Triantafyllidis, 2004) for derivation details):

$$
\begin{aligned}
\mathcal{P}(\mathbf{u}, \mathbf{M}, \widetilde{\mathbf{A}}) & =\int_{V} \rho_{0}\left[\psi(\mathbf{F}, \mathbf{M}, \mathbf{N})-\mu_{0} \mathbf{M} \cdot \widehat{\mathbf{h}}-\mathbf{f} \cdot \mathbf{u}\right] \mathrm{d} V-\int_{\partial V} \mathbf{T} \cdot \mathbf{u} \mathrm{d} S+ \\
& +\int_{\mathbb{R}^{3}} \frac{1}{2 \mu_{0} J}\left[\mathbf{F} \cdot(\boldsymbol{\nabla} \times \widetilde{\mathbf{A}})-\mu_{0} \rho_{0} \mathbf{M}\right] \cdot\left[\mathbf{F} \cdot(\nabla \times \widetilde{\mathbf{A}})-\mu_{0} \rho_{0} \mathbf{M}\right] \mathrm{d} V
\end{aligned}
$$

\footnotetext{
${ }^{1}$ Here $\boldsymbol{\nabla} \equiv \partial() / \partial \mathbf{X}$ denotes the gradient operator in the reference configuration with $\mathbf{x}$ and $\mathbf{X}$ the current and reference position vector respectively of a material point
} 
The potential energy (2.4) is then extremized with respect to $\mathbf{u}, \mathbf{M}$ and $\widetilde{\mathbf{A}}$ to give the strong form of the field equations plus the corresponding boundary/interface conditions. Extremizing the potential energy implies that the first variation of $\mathcal{P}^{2}$ is equal to zero:

$$
\delta \mathcal{P}(\mathbf{u}, \mathbf{M}, \widetilde{\mathbf{A}})=\mathcal{P}_{, \mathbf{u}} \delta \mathbf{u}+\mathcal{P}_{, \mathbf{M}} \delta \mathbf{M}+\mathcal{P}_{, \widetilde{\mathbf{A}}} \delta \widetilde{\mathbf{A}}=0,
$$

and since the variations $\delta \mathbf{u}, \delta \mathbf{M}$ and $\delta \widetilde{\mathbf{A}}$ are arbitrary, each one of the three therms in (2.5) must vanish.

In the following, for continuity of the presentation, we just display the results of the aforementioned variations; for full derivation details the interested reader is referred to Kankanala and Triantafyllidis (2008). Vanishing of the variation of the potential energy $\mathcal{P}$ with respect to displacement $\mathbf{u}$ from (2.5) yields:

$$
\mathcal{P}_{, \mathbf{u}} \delta \mathbf{u}=0 \Rightarrow \boldsymbol{\nabla} \cdot \boldsymbol{\Pi}+\rho_{0} \mathbf{f}=0, \quad \mathbf{X} \in V ; \quad \mathcal{N} \cdot \llbracket \mathbf{\Pi} \rrbracket=\mathbf{T}, \quad \mathbf{X} \in \partial V,
$$

where $\boldsymbol{\Pi}=J \boldsymbol{\sigma} \cdot \mathbf{F}^{-T}$ is the first Piola-Kirchhoff stress (or transpose of the nominal stress) and $\mathcal{N}$ is the unit normal to the boundary $\partial V$ in the reference configuration. Moreover, the Cauchy (or true) stress $\boldsymbol{\sigma}$, is a total stress measure that contains both mechanical and electromagnetic contributions and can be shown to take the form:

$$
\boldsymbol{\sigma}=\rho\left[\frac{\partial \psi}{\partial \mathbf{F}} \cdot \mathbf{F}^{T}+\mu_{0} \mathbf{h M}\right]+\mu_{0}\left[\mathbf{h} \mathbf{h}-\frac{1}{2}(\mathbf{h} \cdot \mathbf{h}) \mathbf{I}\right]
$$

Note that in vacuum (i.e. $\rho=0$ ), the total Cauchy stress is non-zero and equals the Maxwell stress $\mu_{0}[\mathbf{h h}-(1 / 2)(\mathbf{h} \cdot \mathbf{h}) \mathbf{I}]$ as expected from classical electromagnetic theory. The mechanical surface traction $\mathbf{t}$ in the current configuration (counterpart of $\mathbf{T}$ in the reference configuration) can be readily obtained from (2.6) and (2.7) by:

$$
\mathbf{t}=\rho\left[\frac{\partial \psi}{\partial \mathbf{F}} \cdot \mathbf{F}^{T}-\frac{\rho \mu_{0}}{2}(\mathbf{M} \cdot \mathbf{n})^{2} \mathbf{I}\right] \cdot \mathbf{n},
$$

where $\mathbf{n}$ is the unit outward normal to the boundary $\partial V$ in the current configuration.

Next, we consider the vanishing of the variation of the potential energy $\mathcal{P}$ with respect to the magnetization $\mathbf{M}$ according to (2.5), which gives the constitutive relation:

$$
\mathcal{P}_{, \mathbf{M}} \delta \mathbf{M}=0 \Rightarrow \mu_{0} \mathbf{h}=\frac{\partial \psi}{\partial \mathbf{M}}, \quad \mathbf{X} \in V .
$$

Finally from (2.5), vanishing of the variation of the potential energy $\mathcal{P}$ with respect to the perturbed magnetic vector potential $\widetilde{\mathbf{A}}$ results in Ampère's differential equation and boundary/interface condition in the reference configuration:

$$
\mathcal{P}_{, \widetilde{\mathbf{A}}} \delta \widetilde{\mathbf{A}}=0 \Rightarrow \boldsymbol{\nabla} \times \mathbf{H}=\mathbf{0}, \quad \mathbf{X} \in \mathbb{R}^{3} ; \quad \mathcal{N} \times \llbracket \mathbf{H} \rrbracket=\mathbf{0}, \quad \mathbf{X} \in \partial V ; \quad \mathbf{H}=\mathbf{h} \cdot \mathbf{F} .
$$

The pointwise equations and boundary/interface conditions derived from (2.5), govern the behavior of the MRE layer, the non-magnetic substrate as well as the surrounding air.

\subsection{Geometry and loading}

The problem at hand pertains to a MRE layer/non-magnetic substrate assembly under plane strain conditions subjected to combined mechanical and magnetic loading, as shown in Fig. 1.

\footnotetext{
${ }^{2}$ Henceforth, $\mathcal{P}, \mathbf{g} \delta \mathbf{g}$ and $(\mathcal{P}, \mathbf{g g} \delta \mathbf{g}) \Delta \mathbf{g}$ denote, respectively, the first and second Frechet derivatives of the potential energy $\mathcal{P}$ with respect to the independent variables $\mathbf{g} \equiv\{\mathbf{u}, \mathbf{M}, \widetilde{\mathbf{A}}\}$.
} 
a)

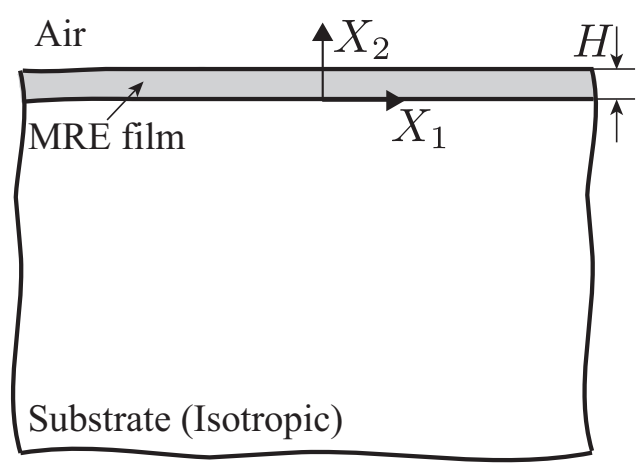

c) MRE film Microstructure

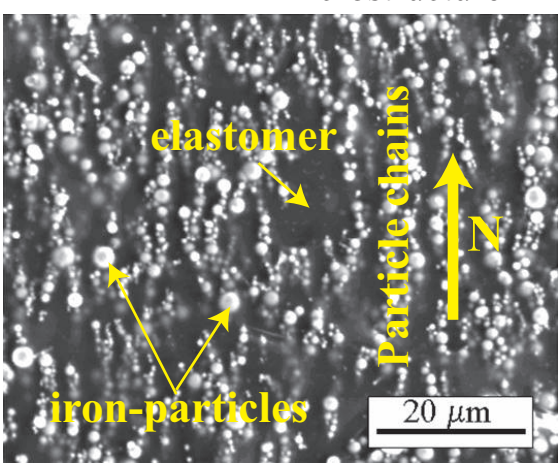

b)

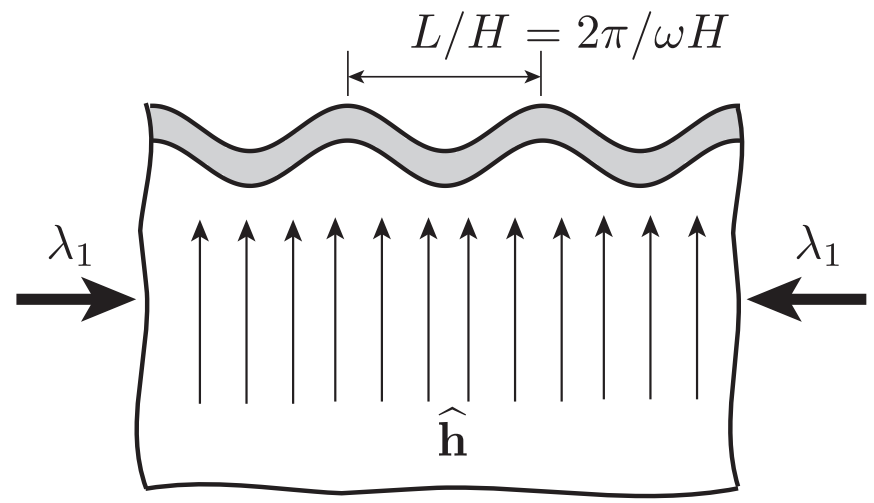

d)
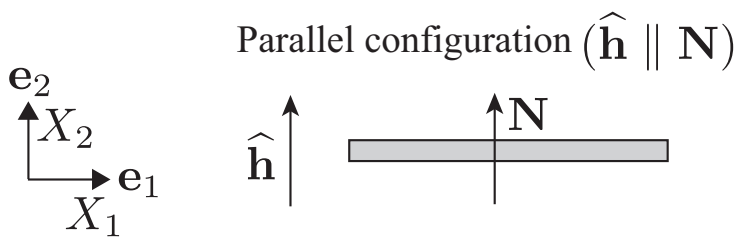

Perpendicular configuration $(\widehat{\mathbf{h}} \perp \mathbf{N})$

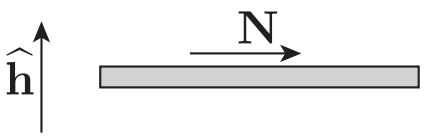

Figure 1: Schematics of the MRE-layer/non-magnetic substrate, plane strain boundary value problem. Part (a) shows the geometry of the stress-free, reference configuration of the system. The perfectly bonded interface is taken for convenience at $X_{2}=0$ and is aligned with the $X_{1}$-axis; the MRE layer has an initial thickness $H$ and lies atop the non-magnetic, semi-infinite substrate. Part (b) shows the deformed, (initial post-bifurcated) configuration under applied mechanical and magnetic loads, i.e., an applied stretch $\lambda_{1}$ along the $X_{1}$ (interface) direction and an applied h-field $\widehat{\mathbf{h}}=\widehat{h} \mathbf{e}_{2}$ along the $X_{2}$ (normal to interface) direction. Part (c) shows an electron micrograph of the anisotropic (orthotropic) MRE layer; the application of a magnetic field during the curing process leads to formation of particle chains aligned with the curing field direction. Part (d), sketches the MRE film and the two different magnetic loadings considered: case i) where the particle chains of direction $\mathbf{N}$ are parallel to the applied h-field $\widehat{\mathbf{h}}$ and case ii) where particle chains of direction $\mathbf{N}$ are perpendicular to the applied h-field $\widehat{\mathbf{h}}$.

More specifically, the stress-free, reference (undeformed) configuration is depicted in Fig. 1a, and consists of an MRE layer of initial thickness $H$ resting atop a non-magnetic semi-infinite substrate. The perfectly bonded interface is taken for convenience at $X_{2}=0$ and is aligned with the $X_{1}$-axis. The Lagrangian formulation of the governing equations used in this work and the assumption of (finite) plane strain deformations dictate that all field quantities are functions of $\left(X_{1}, X_{2}\right)$, the reference cartesian coordinates of each material point.

The layered structure is subjected to three different loadings. The first loading case is purely magnetic and consists in an applied magnetic field along the $X_{2}$ direction, i.e. normal to the interface, while at the same time imposing zero strain at the interface ${ }^{3}\left(\lambda_{1}=1\right)$ along the $X_{1}$ direction during this loading process. The second loading case is purely mechanical and consists of compressing the interface along the $X_{1}$ direction by $\lambda_{1}<1$. The third loading case consists of a combination of the previous two: applying

\footnotetext{
${ }^{3}$ Here $\lambda_{i}$ with $i=1,2$ denote the principal stretch ratios in each layer; perfect bonding dictates that the imposed $\lambda_{1}$ is the same for both MRE layer and non-magnetic substrate; as expected $\lambda_{2}$ is different at the layer and substrate, depending on applied loading and constitutive response.
} 
compression along the $X_{1}$ direction, keeping $\lambda_{1}<1$ constant, and subsequently increasing magnetic field $\widehat{\mathbf{b}}=\mu_{0} \widehat{\mathbf{h}}$ along the $X_{2}$ direction. It should be noted that due to the independence of the strain energy on the sign of the magnetization, the sign of the applied magnetic field does not affect the solution of the problem at hand and hence is always directed along the positive $X_{2}$ direction. Of interest are the conditions at the onset of bifurcation in the substrate/layer assembly, which will be investigated in the next section. A schematic of the deformed, (initial post-bifurcated) configuration is depicted in Fig. 1b. The $X_{1}$-invariance of the principal solution, allows a Fourier decomposition of the eigenmode; the wavelength $L$ and corresponding wavenumber $\omega$ are also sketched in Fig. 1b.

The MRE layers used in this study are those investigated in Danas et al. (2012), which due to their manufacturing process (curing in the presence of a magnetic field) are orthotropic, as seen in the micrograph in Fig. 1c. Due to the MRE layer orthotropy, two different layer configurations will be considered, as depicted in Fig. 1d; the parallel configuration $(\widehat{\mathbf{h}} \| \mathbf{N})$ where the particle chains of direction $\mathbf{N}$ are set parallel to the applied h-field $\widehat{\mathbf{h}}$ and the perpendicular configuration $(\widehat{\mathbf{h}} \perp \mathbf{N})$ where particle chains of direction $\mathbf{N}$ are set perpendicular to the applied h-field $\widehat{\mathbf{h}}$.

\subsection{Material selection}

The MRE layer considered in this study has been presented in Danas et al. (2012) and consists of nearly spherical carbonyl iron particles (ISP, grade S-3700) embedded in natural rubber, i.e. cispolyisoprene, whereas the particle sizes range from approximately $0.5 \mu \mathrm{m}$ to $5 \mu \mathrm{m}$ and have a volume fraction of $25 \%$. These composites are manufactured in the presence of a curing magnetic field and their iron particles form chain-like structures whose direction is described by the unit vector $\mathbf{N}$, as shown in Fig. 1c. Consequently, the coupled magneto-mechanical response of the MRE layer is described by a transversely isotropic energy density function $\psi_{l}(\mathbf{F}, \mathbf{M}, \mathbf{N})$ which depends on the deformation gradient tensor $\mathbf{F}$, the specific magnetization $\mathbf{M}$ and the reference configuration particle-chain orientation vector $\mathbf{N}$, as discussed in (2.1). For such transversely isotropic materials, their energy density is a function of ten independent invariants involving, F, M and N. In the present case, following Danas et al. (2012), we will make use of only eight of these ten independent invariants ${ }^{4}$ given by:

$$
\begin{array}{lll}
I_{1} \equiv \operatorname{Tr}(\mathbf{F} \cdot \mathbf{F}), & I_{3} \equiv J^{2}=(\operatorname{det} \mathbf{F})^{2}, & I_{4} \equiv \mathbf{N} \cdot \mathbf{F}^{T} \cdot \mathbf{F} \cdot \mathbf{N} \\
I_{6} \equiv \mathbf{M} \cdot \mathbf{M}, & I_{7} \equiv \mathbf{M} \cdot \mathbf{F} \cdot \mathbf{F}^{T} \cdot \mathbf{M}, & I_{8} \equiv \mathbf{M} \cdot\left(\mathbf{F} \cdot \mathbf{F}^{T}\right)^{2} \cdot \mathbf{M}, \\
I_{9} \equiv(\mathbf{M} \cdot \mathbf{F} \cdot \mathbf{N})^{2}, & I_{10} \equiv(\mathbf{M} \cdot \mathbf{F} \cdot \mathbf{N})\left(\mathbf{M} \cdot \mathbf{F} \cdot \mathbf{F}^{T} \cdot \mathbf{F} \cdot \mathbf{N}\right) .
\end{array}
$$

Consequently, the transversely isotropic energy density function of the MRE layer $\psi_{l}$ takes the form ${ }^{5}$ :

$$
\begin{aligned}
\rho_{0} \psi_{l}(\mathbf{F}, \mathbf{M}, \mathbf{N}) & =\frac{G_{l}^{\prime}}{2}(J-1)^{2}+\frac{G_{l}}{2}\left[C_{1} \sum_{k=1}^{5} d_{1 k}\left(J^{-2 / 3} I_{1}-3\right)^{k}+C_{4} \sum_{k=2}^{4} d_{4 k}\left(J^{-2 / 3} I_{4}-1\right)^{k}+\right. \\
& +C_{6} \frac{I_{6}}{M_{s}^{2}}+C_{6}^{*}\left\{\frac{1}{2} \ln \left[1-\left(\frac{I_{6}}{M_{s}^{2}}\right)^{2}\right]+\frac{I_{6}}{M_{s}^{2}} \tanh ^{-1}\left(\frac{I_{6}}{M_{s}^{2}}\right)\right\}+ \\
& \left.+C_{7} \frac{I_{7}}{M_{s}^{2}}+C_{8} \frac{I_{8}}{M_{s}^{2}}+C_{9} \frac{I_{9}}{M_{s}^{2}}+C_{10} \frac{I_{10}}{M_{s}^{2}}\right]
\end{aligned}
$$

with $G_{l}=1.0374 \mathrm{MPa}$ denoting the shear modulus of the MRE layer associated with shearing perpendicular to the particle chains and $G_{l}^{\prime}$ being the Lamé constant associated with the compressible part of the energy taken in this study large compared to $G_{l}$, i.e. $G_{l}^{\prime}=100 G_{l}$. The magnetization at saturation $M_{s}$ is such that $\mu_{0}\left(\rho_{0} M_{s}\right)_{l}=0.45 T$. The numerical values of the material constants in $(2.12)$ are:

$$
\begin{aligned}
& C_{1}=1, \quad d_{11}=1, \quad d_{12}=-7, \quad d_{13}=120, \quad d_{14}=-700, \quad d_{15}=3000, \\
& C_{4}=0.1, \quad d_{42}=1, \quad d_{43}=-21, \quad d_{44}=90, \\
& C_{6}=0.36, \quad C_{7}=-0.32, \quad C_{8}=0.11, \quad C_{9}=-0.12, \quad C_{10}=0.08, \quad C_{6}^{*}=0.1 .
\end{aligned}
$$

\footnotetext{
${ }^{4}$ The invariants $I_{2} \equiv 0.5\left[\left(I_{1}\right)^{2}-\operatorname{Tr}\left(\left(\mathbf{F}^{T} \cdot \mathbf{F}\right)^{2}\right)\right]$ and $I_{5} \equiv \mathbf{N} \cdot\left(\mathbf{F} \cdot \mathbf{F}^{T}\right)^{2} \cdot \mathbf{N}$ have been omitted from the above equations since they are not necessary in constructing a satisfactory, experimentally-based energy density function.

${ }^{5}$ Note that the energy function used in this study has been appropriately modified to include compressibility effects, in contrast to the incompressible model in Danas et al. (2012).
} 
The substrate is described for simplicity by a purely mechanical, compressible, isotropic Neo-Hookean model, whose energy function reads:

$$
\rho_{0} \psi_{s}(\mathbf{F})=\frac{G_{s}}{2}\left(J^{-2 / 3} I_{1}-3\right)+\frac{G_{s}^{\prime}}{2}(J-1)^{2},
$$

where $G_{s}$ and $G_{s}^{\prime}=100 G_{s}$ are its shear modulus and Lamé constants, respectively. It should be noted that the reference configuration density of the substrate is taken for simplicity equal to that of the MRE layer.

\subsection{Principal solution}

In this section, we describe the principal solution of the MRE substrate/layer assembly, which due to the material's orthotropy and homogeneity as well as the corresponding symmetry of the applied loading consists of a constant strain state, i.e. constant stretches $\left(\lambda_{1}, \lambda_{2}\right)$, in each layer and a constant magnetization $M_{2}$ for the MRE layer.

Due to continuity of the magnetic field $\mathbf{b}$ across the substrate/layer interface (i.e. $\mathbf{b}=\widehat{\mathbf{b}}$ and $\widetilde{\mathbf{b}}=\mathbf{0}$ for the MRE layer, the substrate and the air), the deformation gradient $\mathbf{F}$, the total magnetic field $\mathbf{b}$ and the magnetization $\mathbf{M}$ of the MRE layer take the form:

$$
\mathbf{F}=\lambda_{1} \mathbf{e}_{1} \mathbf{e}_{1}+\lambda_{2} \mathbf{e}_{2} \mathbf{e}_{2}, \quad \mathbf{b}=\mu_{0} \widehat{h} \mathbf{e}_{2}, \quad \mathbf{M}=M_{2} \mathbf{e}_{2} .
$$

For a given $\lambda_{1}$ and $\widehat{h}$ one can find $\lambda_{2}$ and $M_{2}$ in the MRE layer by exploiting the fact that the mechanical traction $t_{2}$ as well as the magnetic field perturbation $\widetilde{b}_{2}$ both vanish, namely:

$$
t_{2}\left(\lambda_{2}, M_{2}\right)=0, \quad \widetilde{b}_{2}\left(\lambda_{2}, M_{2}\right)=\mu_{0}\left(h_{2}-\widehat{h}+\frac{\rho_{0}}{J} M_{2}\right)=0 .
$$

In the above system of nonlinear algebraic equations the mechanical traction component $t_{2}$ is calculated with the help of (2.8) and the layer's energy density (2.12), while the h-field component $h_{2}$ is calculated with the help of (2.9) and again the layer's energy density (2.12). A Newton-Raphson numerical method is employed to solve the above nonlinear system for two equations for the two unknowns, since no easy analytical solution can be found due to the complexity of the adopted expressions for the layer's energy density $\psi_{l}$ in $(2.12)$.

For the principal solution of the non-magnetic substrate solution, one only needs to find $\lambda_{2}$ in terms of the applied stretch at the interface $\lambda_{1}$. The corresponding equation is the vanishing of the traction $t_{2}=0$ at the interface between the layer and the substrate. Using the expression for the traction in (2.8) and the substrate energy density $\psi_{s}$ from (2.14), the stretch $\lambda_{2}$ in the substrate can be expressed in terms of the interface stretch $\lambda_{1}$ by the following analytical expression:

$$
t_{2}\left(\lambda_{2}\right)=0 \Rightarrow \lambda_{2}=\left\{G_{s}^{\prime} \lambda_{1}+\left[\left(G_{s}^{\prime} \lambda_{1}\right)^{2}+4 G_{s} G_{s}^{\prime}\left(\lambda_{1}\right)^{2}+4\left(G_{s}\right)^{2}\right]^{1 / 2}\right\} /\left\{2\left[G_{s}^{\prime}\left(\lambda_{1}\right)^{2}+G_{s}\right]\right\} .
$$

The non-magnetic nature of the substrate dictates $\mathbf{M}=\mathbf{0}$ (same holds for the air); note however that the magnetic and h-fields in the substrate, as well as in the air are non-zero and can easily be found from continuity of the transverse component of the magnetic field, i.e. $b_{2}=\mu_{0} \widehat{h}$.

\section{Onset of bifurcation}

Of interest here is the stability - and more precisely the onset of a bifurcation instability - in the principal solution, presented in the previous section and obtained by extremizing the potential energy $\mathcal{P}$ in (2.4) with respect to the three independent fields, denoted compactly by $\mathbf{g} \equiv\{\mathbf{u}, \mathbf{M}, \widetilde{\mathbf{A}}\}$. This solution depends on the applied mechanical and magnetic loading, which is conveniently denoted henceforth using the load vector $\boldsymbol{\Lambda} \equiv\left\{\lambda_{1}, \widehat{h}\right\}$. Although there is no unique way of applying such a loading, the principal solution is independent of the loading history since the problem is conservative.

At relatively small values of the applied $\operatorname{load} \boldsymbol{\Lambda}$, the principal solution $\mathbf{g}_{0}(\boldsymbol{\Lambda})$ of $(2.5)$ is stable, i.e. it is a local minimizer of the potential energy satisfying $\left(\mathcal{P}, \mathbf{g g}_{0}\left(\mathbf{g}_{0}(\boldsymbol{\Lambda}), \boldsymbol{\Lambda}\right) \delta \mathbf{g}\right) \delta \mathbf{g}>0$, for arbitrary perturbations $\delta \mathbf{g} \neq 0$. As the applied load increases, it reaches a critical load $\boldsymbol{\Lambda}_{c}=\left\{\lambda_{1}^{c}, \widehat{h}_{c}\right\}$ where the principal solution at hand $\mathbf{g}_{0}\left(\boldsymbol{\Lambda}_{c}\right)$ is no longer a local minimizer. At this point, the energy vanishes along a particular direction $\Delta \mathbf{g}$, called the critical mode, which satisfies the condition:

$$
\left(\mathcal{P}, \mathbf{g g}\left(\mathbf{g}_{0}\left(\boldsymbol{\Lambda}_{c}\right), \boldsymbol{\Lambda}_{c}\right) \Delta \mathbf{g}\right) \delta \mathbf{g}=0
$$


Solving the above-stated eigenvalue problem, requires the consideration of the second Frechet derivatives of the potential energy $\mathcal{P}$ in (2.4) with respect to the independent variables $(\mathbf{u}, \mathbf{M}, \widetilde{\mathbf{A}})$. Due to the plane-strain character of the problem, $\mathbf{u}=u_{1} \mathbf{e}_{1}+u_{2} \mathbf{e}_{2}, \mathbf{M}=M_{1} \mathbf{e}_{1}+M_{2} \mathbf{e}_{2}$ for the MRE layer and $\widetilde{\mathbf{A}}=\widetilde{A}_{3} \mathbf{e}_{3}$. For convenience, we denote $\widetilde{A}_{3} \equiv \alpha$. Using the independence of $(\delta \mathbf{u}, \delta \mathbf{M}, \delta \alpha)$, the eigenvalue problem stated in $(3.1)$ can be written as ${ }^{6}$ :

$$
\begin{aligned}
&\left(\mathcal{P}_{, \mathbf{g u}} \Delta \mathbf{g}\right) \delta \mathbf{u}=0 \quad \Rightarrow \quad \int_{V}\left[\mathcal{L}_{i j k l}^{u u} \Delta u_{k, l}+\mathcal{L}_{i j k}^{u M} \Delta M_{k}+\mathcal{L}_{i j k}^{u \alpha} \Delta \alpha, k\right] \delta u_{i, j} \mathrm{~d} V=0, \\
&\left(\mathcal{P}_{, \mathbf{g M}} \Delta \mathbf{g}\right) \delta \mathbf{M}=0 \quad \Rightarrow \quad \int_{V}\left[\mathcal{L}_{i j k}^{M u} \Delta u_{j, k}+\mathcal{L}_{i j}^{M M} \Delta M_{j}+\mathcal{L}_{i j}^{M \alpha} \Delta \alpha_{, j}\right] \delta M_{i} \mathrm{~d} V=0, \\
&\left(\mathcal{P}_{, \mathbf{g} \alpha} \Delta \mathbf{g}\right) \delta \alpha=0 \quad \Rightarrow \quad \int_{\mathbb{R}^{2}}\left[\mathcal{L}_{i j k}^{\alpha u} \Delta u_{j, k}+\mathcal{L}_{i j}^{\alpha M} \Delta M_{j}+\mathcal{L}_{i j}^{\alpha \alpha} \Delta \alpha_{, j}\right] \delta a_{, i} \mathrm{~d} V=0,
\end{aligned}
$$

where the coefficients in (3.2) are detailed in Appendix A. The Euler-Lagrange (point-wise) differential equations of (3.2) can be obtained through standard integration by parts, leading to:

$$
\begin{array}{cc}
{\left[\mathcal{L}_{i j k l}^{u u} \Delta u_{k, l}+\mathcal{L}_{i j k}^{u M} \Delta M_{k}+\mathcal{L}_{i j k}^{u \alpha} \Delta \alpha_{, k}\right]_{, j}=0,} & \mathbf{X} \in V \\
\mathcal{L}_{i j k}^{M u} \Delta u_{j, k}+\mathcal{L}_{i j}^{M M} \Delta M_{j}+\mathcal{L}_{i j}^{M \alpha} \Delta \alpha_{, j}=0, & \mathbf{X} \in V \\
{\left[\mathcal{L}_{i j k}^{\alpha u} \Delta u_{j, k}+\mathcal{L}_{i j}^{\alpha M} \Delta M_{j}+\mathcal{L}_{i j}^{\alpha \alpha} \Delta \alpha_{, j}\right]_{, i}=0,} & \mathbf{X} \in \mathbb{R}^{2}
\end{array}
$$

where $V$ is the domain occupied by the solids (layer and substrate). These differential equations need to be supplemented with the appropriate natural boundary/interface conditions at the MRE substrate/layer interface and the MRE layer/air boundary, which from (3.2) are found to be:

$$
\begin{array}{lll}
\left\{\mathcal{L}_{i 2 k l}^{u u} \Delta u_{k, l}+\mathcal{L}_{i 2 k}^{u M} \Delta M_{k}+\mathcal{L}_{i 2 k}^{u \alpha} \Delta \alpha_{, k}\right\}_{l}=\left\{\mathcal{L}_{i 2 k l}^{u u} \Delta u_{k, l}+\mathcal{L}_{i 2 k}^{u M} \Delta M_{k}+\mathcal{L}_{i 2 k}^{u \alpha} \Delta \alpha_{, k}\right\}_{s}, & \left(X_{1}, X_{2}\right) \in \mathbb{R} \times\{0\} \\
\left\{\mathcal{L}_{2 j k}^{\alpha u} \Delta u_{j, k}+\mathcal{L}_{2 j}^{\alpha M} \Delta M_{j}+\mathcal{L}_{2 j}^{\alpha \alpha} \Delta \alpha_{, j}\right\}_{l}=\left\{\mathcal{L}_{2 j k}^{\alpha u} \Delta u_{j, k}+\mathcal{L}_{2 j}^{\alpha M} \Delta M_{j}+\mathcal{L}_{2 j}^{\alpha \alpha} \Delta \alpha_{, j}\right\}_{s}, & \left(X_{1}, X_{2}\right) \in \mathbb{R} \times\{0\} \\
\left\{\mathcal{L}_{i 2 k l}^{u u} \Delta u_{k, l}+\mathcal{L}_{i 2 k}^{u M} \Delta M_{k}+\mathcal{L}_{i 2 k}^{u \alpha} \Delta \alpha_{, k}\right\}_{l}=0, & \left(X_{1}, X_{2}\right) \in \mathbb{R} \times\{H\} \\
\left\{\mathcal{L}_{2 j k}^{\alpha u} \Delta u_{j, k}+\mathcal{L}_{2 j}^{\alpha M} \Delta M_{j}+\mathcal{L}_{2 j}^{\alpha \alpha} \Delta \alpha_{, j}\right\}_{l}=0, & \left(X_{1}, X_{2}\right) \in \mathbb{R} \times\{H\} .
\end{array}
$$

To the above natural boundary/interface conditions, one must also add the essential boundary/interface conditions which reflect the continuity of displacement and magnetic perturbation potential, namely:

$$
\begin{array}{ll}
\left\{\Delta u_{i}\right\}_{l}=\left\{\Delta u_{i}\right\}_{s}, & \left(X_{1}, X_{2}\right) \in \mathbb{R} \times\{0\} \\
\{\Delta \alpha\}_{l}=\{\Delta \alpha\}_{s}, & \left(X_{1}, X_{2}\right) \in \mathbb{R} \times\{0\} \\
\{\Delta \alpha\}_{l}=\{\Delta \alpha\}_{a}, & \left(X_{1}, X_{2}\right) \in \mathbb{R} \times\{H\} .
\end{array}
$$

In expressions (3.4) and (3.5), the subscripts $l, s$ and $a$ denote the MRE layer, substrate and air, respectively.

\subsection{Euler-Lagrange equations for the MRE layer}

By expressing $\Delta \mathbf{M}$ in terms of $\Delta \mathbf{u}, \Delta \alpha$ from $(3.3)_{2}$, the system of Euler-Lagrange equations for the MRE layer given by $(3.3)_{1}$ and $(3.3)_{3}$, can be recast in the form:

$$
\left.\begin{array}{c}
{\left[L_{i j k l}^{u u} \Delta u_{k, l}+L_{i j k}^{u \alpha} \Delta \alpha_{, k}\right]_{, j}=0} \\
{\left[L_{i j k}^{\alpha u} \Delta u_{j, k}+L_{i j}^{\alpha \alpha} \Delta \alpha_{, j}\right]_{, i}=0}
\end{array}\right\},\left(X_{1}, X_{2}\right) \in \mathbb{R} \times[0, H] .
$$

\footnotetext{
${ }^{6}$ Henceforth all Latin indexes range in value from 1 to 2 and Einstein's summation convention is implied over repeated indexes.
} 
The coefficients appearing in (3.6) are defined in terms of the coefficients introduced in (3.2) by:

$$
\begin{aligned}
& L_{i j k l}^{u u} \equiv \mathcal{L}_{i j k l}^{u u}-\mathcal{L}_{i j p}^{u M}\left(\mathcal{L}_{p q}^{M M}\right)^{-1} \mathcal{L}_{q k l}^{M u}, \\
& L_{i j k}^{u \alpha} \equiv L_{k i j}^{\alpha u}=\mathcal{L}_{i j k}^{u \alpha}-\mathcal{L}_{i j p}^{u M}\left(\mathcal{L}_{p q}^{M M}\right)^{-1} \mathcal{L}_{q k}^{M \alpha}, \\
& L_{i j}^{\alpha \alpha} \equiv \mathcal{L}_{i j}^{\alpha \alpha}-\mathcal{L}_{i p}^{\alpha M}\left(\mathcal{L}_{p q}^{M M}\right)^{-1} \mathcal{L}_{q j}^{M \alpha} .
\end{aligned}
$$

The solution to system (3.6) of differential equations with constant coefficients, can be put in the form:

$$
\begin{aligned}
& \Delta u_{j}\left(X_{1}, X_{2}\right)=\exp \left(i \omega X_{1}\right)\left[\sum_{I} \Delta U_{j}^{I} \exp \left(\zeta_{I} \omega X_{2}\right)\right], \\
& \Delta \alpha\left(X_{1}, X_{2}\right)=\exp \left(i \omega X_{1}\right)\left[\sum_{I} \Delta \mathcal{A}^{I} \exp \left(\zeta_{I} \omega X_{2}\right)\right],
\end{aligned}
$$

where the maximum value of integer $I$, which represents the number of the system's independent eigenmodes, will be specified subsequently. Substitution of expressions (3.8) in (3.6) leads to the following algebraic eigenvalue problem for $\zeta_{I}$ and the corresponding amplitudes $\left(\Delta U_{1}^{I}, \Delta U_{2}^{I}, \Delta \mathcal{A}^{I}\right)$ :

$$
\underbrace{\left[\begin{array}{ccc}
-L_{1111}^{u u}+\left(\zeta_{I}\right)^{2} L_{1212}^{u u} & i \zeta_{I}\left(L_{1122}^{u u}+L_{1221}^{u u}\right) & -L_{111}^{u \alpha}+\left(\zeta_{I}\right)^{2} L_{122}^{u \alpha} \\
i \zeta_{I}\left(L_{2112}^{u u}+L_{2211}^{u u}\right) & -L_{2121}^{u u}+\left(\zeta_{I}\right)^{2} L_{2222}^{u u} & i \zeta_{I}\left(L_{212}^{u \alpha}+L_{221}^{u \alpha}\right) \\
-L_{111}^{\alpha u}+\left(\zeta_{I}\right)^{2} L_{212}^{\alpha u} & i \zeta_{I}\left(L_{122}^{\alpha u}+L_{221}^{\alpha u}\right) & -L_{11}^{\alpha \alpha}+\left(\zeta_{I}\right)^{2} L_{22}^{\alpha \alpha}
\end{array}\right]}_{\mathbf{Q}_{l}\left(\zeta_{I}\right)} \underbrace{\left\{\begin{array}{c}
\Delta U_{1}^{I} \\
\Delta U_{2}^{I} \\
\Delta \mathcal{A}^{I}
\end{array}\right\}}_{\Delta \mathbf{U}_{l}^{I}}=\left\{\begin{array}{c}
0 \\
0 \\
0
\end{array}\right\} .
$$

In the above expression, $\zeta_{I}$ are the six roots of the bi-cubic characteristic equation, which implies that the integer $I$ ranges from 1 to 6 :

$$
\operatorname{det} \mathbf{Q}_{l}\left(\zeta_{I}\right)=0 ; \quad 1 \leq I \leq 6
$$

For each root $\zeta_{I}$ in (3.10), one can find from (3.9) the linear relationship between the corresponding $\Delta U_{j}^{I}$ and $\Delta \mathcal{A}^{I}$ :

$$
\Delta U_{j}^{I}=\Delta \mathcal{U}_{j}^{I} \Delta \mathcal{A}^{I} ; \quad 1 \leq j \leq 2, \quad 1 \leq I \leq 6,
$$

where the coefficients $\Delta \mathcal{U}_{i}^{I}$, introduced for convenience in notation, are functions of the constants $\boldsymbol{L}^{\boldsymbol{u} u}$, $\boldsymbol{L}^{\boldsymbol{u \alpha}}, \boldsymbol{L}^{\boldsymbol{\alpha u}}, \boldsymbol{L}^{\boldsymbol{\alpha} \boldsymbol{\alpha}}$ defined in (3.7).

\subsection{Euler-Lagrange equations for the substrate}

The system of equations for the substrate simplifies significantly since it has no magnetic-mechanical coupling. Consequently from (3.3), we obtain the following decoupled system of Euler-Lagrange equations:

$$
\left.\begin{array}{c}
{\left[\mathcal{L}_{i j k l}^{u u} \Delta u_{k, l}\right]_{, j}=0} \\
{\left[\mathcal{L}_{i j}^{\alpha \alpha} \Delta \alpha_{, j}\right]_{, i}=0}
\end{array}\right\},\left(X_{1}, X_{2}\right) \in \mathbb{R} \times(-\infty, 0] .
$$

The solution to system (3.12) of differential equations with constant coefficients is given by:

$$
\begin{aligned}
& \Delta u_{j}\left(X_{1}, X_{2}\right)=\exp \left(i \omega X_{1}\right)\left[\sum_{I} \Delta V_{j}^{I} \exp \left(\xi_{I} \omega X_{2}\right)\right], \\
& \Delta \alpha\left(X_{1}, X_{2}\right)=\exp \left(i \omega X_{1}\right)\left[\Delta \mathcal{A}_{s} \exp \left(\theta_{s} \omega X_{2}\right)\right]
\end{aligned}
$$

where again the maximum value of integer $I$ is determined below.

For the $\Delta \mathbf{u}$ component of the eigenmode, substitution of expressions $(3.13)_{1}$ in $(3.12)_{1}$ leads to the following algebraic eigenvalue problem for $\xi_{I}$ and the corresponding amplitudes $\left(\Delta V_{1}^{I}, \Delta V_{2}^{I}\right)$ :

$$
\underbrace{\left[\begin{array}{cc}
-\mathcal{L}_{1111}^{u u}+\left(\xi_{I}\right)^{2} \mathcal{L}_{1212}^{u u} & i \xi_{I}\left(\mathcal{L}_{1122}^{u u}+\mathcal{L}_{1221}^{u u}\right) \\
i \xi_{I}\left(\mathcal{L}_{2112}^{u u}+\mathcal{L}_{2211}^{u u}\right) & -\mathcal{L}_{2121}^{u u}+\left(\xi_{I}\right)^{2} \mathcal{L}_{2222}^{u u}
\end{array}\right]}_{\mathbf{Q}_{s}\left(\xi_{I}\right)} \underbrace{\left\{\begin{array}{c}
\Delta V_{1}^{I} \\
\Delta V_{2}^{I}
\end{array}\right\}}_{\mathbf{\Delta} \mathbf{V}_{s}^{I}}=\left\{\begin{array}{c}
0 \\
0
\end{array}\right\} .
$$


In the above expression, $\xi_{I}$ are the two solutions with positive real part of the bi-quadratic characteristic equation, which implies that $I$ ranges from 1 to 2 :

$$
\operatorname{det} \mathbf{Q}_{s}\left(\xi_{I}\right)=0 ; \quad \mathcal{R} e\left(\xi_{I}\right)>0, \quad 1 \leq I \leq 2 .
$$

Out of the four (in general complex) roots of the above bi-quadratic equation only the two with positive real part are considered, for they need to satisfy the decay condition for the eigenmode $\Delta \mathbf{u} \rightarrow 0$ as $X_{2} \rightarrow-\infty$. For each root $\xi_{I}$ of (3.15), one can find from (3.14) the linear relationship between $\Delta V_{1}^{I}$ and $\Delta V_{2}^{I}$, namely:

$$
\Delta V_{1}^{I}=\Delta \mathcal{V}_{1}^{I} \Delta V_{2}^{I} ; \quad 1 \leq I \leq 2,
$$

where the coefficients $\Delta \mathcal{V}_{1}^{I}$, introduced for convenience of notation, are functions of the constants $\mathcal{L}^{\boldsymbol{u} u}$ defined in (3.2).

For the independent $\Delta \alpha$ component of the eigenmode, substitution of expression $(3.13)_{2}$ into $(3.12)_{2}$ leads to the following algebraic equation relating $\theta_{s}$ to the corresponding amplitude $\Delta \mathcal{A}_{s}$ :

$$
\left[-\mathcal{L}_{11}^{\alpha \alpha}+\left(\theta_{s}\right)^{2} \mathcal{L}_{22}^{\alpha \alpha}\right] \Delta \mathcal{A}_{s}=0, \quad \theta_{s}=\left[\mathcal{L}_{11}^{\alpha \alpha} / \mathcal{L}_{22}^{\alpha \alpha}\right]^{1 / 2}
$$

Similarly, out of the two real roots of the above equation (it is easily shown that $\mathcal{L}_{11}^{\alpha \alpha} / \mathcal{L}_{22}^{\alpha \alpha}>0$ ), only the positive root is considered in order to satisfy decay condition $\Delta \alpha \rightarrow 0$ of the eigenmode as $X_{2} \rightarrow-\infty$.

\subsection{Euler-Lagrange equations for the air}

The system of equations for the air is even simpler than the substrate's, since only the magnetic field equations $(3.3)_{3}$ survive:

$$
\left[\mathcal{L}_{i j}^{\alpha \alpha} \Delta \alpha_{, j}\right]_{, i}=0, \quad\left(X_{1}, X_{2}\right) \in \mathbb{R} \times[H, \infty) .
$$

As before, we have the following representation for the eigenmode $\Delta \alpha(\mathbf{X})$, solution of the constant coefficient equation (3.18):

$$
\Delta \alpha\left(X_{1}, X_{2}\right)=\exp \left(i \omega X_{1}\right)\left[\Delta \mathcal{A}_{a} \exp \left(\theta_{a} \omega X_{2}\right)\right] .
$$

Substitution of expression (3.19) into (3.18) leads to the following algebraic relation between $\theta_{a}$ and the corresponding amplitude $\Delta \mathcal{A}_{a}$ :

$$
\left[-\mathcal{L}_{11}^{\alpha \alpha}+\left(\theta_{a}\right)^{2} \mathcal{L}_{22}^{\alpha \alpha}\right] \Delta \mathcal{A}_{a}=0, \quad \theta_{a}=-\left[\mathcal{L}_{11}^{\alpha \alpha} / \mathcal{L}_{22}^{\alpha \alpha}\right]^{1 / 2}
$$

where again one can easily show that $\mathcal{L}_{11}^{\alpha \alpha} / \mathcal{L}_{22}^{\alpha \alpha}>0$. This time the negative root is considered in order to satisfy decay condition $\Delta \alpha \rightarrow 0$ for the eigenmode as $X_{2} \rightarrow \infty$.

Thus far, in the above three subsections, we have constructed the general solution for the EulerLagrange equations of the eigenmode, stated in (3.3). This information is needed to determine the full eigenvalue problem for the system at hand, which is the object of the next subsection.

\subsection{Determination of critical loads and corresponding eigenmodes}

To complete the onset of bifurcation analysis and fully determine the critical loads and corresponding eigenmodes, we must also use the remaining information on the system, namely the natural and essential boundary/interface conditions.

To this end, recalling from (3.4) and (3.5) the natural and essential interface conditions at the $\mathrm{MRE} /$ substrate interface and taking also into account the orthotropy of the system, we obtain:

$$
\left.\begin{array}{r}
\left\{L_{1212}^{u u} \Delta u_{1,2}+L_{1221}^{u u} \Delta u_{2,1}+L_{122}^{u \alpha} \Delta \alpha_{, 2}\right\}_{l}=\left\{\mathcal{L}_{1212}^{u u} \Delta u_{1,2}+\mathcal{L}_{1221}^{u u} \Delta u_{2,1}\right\}_{s} \\
\left\{L_{2211}^{u u} \Delta u_{1,1}+L_{222}^{u u} \Delta u_{2,2}+L_{221}^{u \alpha} \Delta \alpha_{, 1}\right\}_{l}=\left\{\mathcal{L}_{2211}^{u u} \Delta u_{1,1}+\mathcal{L}_{2222}^{u u} \Delta u_{2,2}\right\}_{s} \\
\left\{L_{212}^{\alpha u} \Delta u_{1,2}+L_{221}^{\alpha u} \Delta u_{2,1}+L_{22}^{\alpha \alpha} \Delta \alpha_{, 2}\right\}_{l}=\left\{\mathcal{L}_{22}^{\alpha \alpha} \Delta \alpha_{, 2}\right\}_{s} \\
\left\{\Delta u_{1}\right\}_{l}=\left\{\Delta u_{1}\right\}_{s} \\
\left\{\Delta u_{2}\right\}_{l}=\left\{\Delta u_{2}\right\}_{s} \\
\{\Delta \alpha\}_{l}=\{\Delta \alpha\}_{s}
\end{array}\right\},\left(X_{1}, X_{2}\right) \in \mathbb{R} \times\{0\} .
$$


Similarly, recalling from (3.4) and (3.5) the natural and essential boundary conditions at the MRE surface and taking also into account the orthotropy of the system, we additionally get:

$$
\left.\begin{array}{r}
\left\{L_{1212}^{u u} \Delta u_{1,2}+L_{1221}^{u u} \Delta u_{2,1}+L_{122}^{u \alpha} \Delta \alpha_{, 2}\right\}_{l}=0 \\
\left\{L_{2211}^{u u} \Delta u_{1,1}+L_{2222}^{u u} \Delta u_{2,2}+L_{221}^{u \alpha} \Delta \alpha_{, 1}\right\}_{l}=0 \\
\left\{L_{212}^{\alpha u} \Delta u_{1,2}+L_{221}^{\alpha u} \Delta u_{2,1}+L_{22}^{\alpha \alpha} \Delta \alpha_{, 2}\right\}_{l}=\left\{\mathcal{L}_{22}^{\alpha \alpha} \Delta \alpha\right\}_{a} \\
\{\Delta \alpha\}_{l}=\{\Delta \alpha\}_{a}
\end{array}\right\},\left(X_{1}, X_{2}\right) \in \mathbb{R} \times\{H\} .
$$

Noting that in the MRE layer $\Delta U_{i}^{I}(i=1,2)$ is written in terms of the $\Delta \mathcal{A}^{I}$, and in the substrate $\Delta V_{1}^{I}$ is written in terms of the $\Delta V_{2}^{I}$, and substituting the eigenmode expressions for each layer according to (3.8), (3.13) and (3.19) into the interface/boundary conditions (3.21) and (3.22), we recover a $10 \times 10$ linear system, written compactly in the form:

$$
\sum_{q=1}^{10} \mathcal{D}_{p q}(\boldsymbol{\Lambda}, \omega H) \mathcal{H}_{q}=0, \quad 1 \leq p \leq 10
$$

where the 10 -dimensional vector of unknowns $\mathcal{H}$ is defined by:

$$
\mathcal{H} \equiv\left\{\left[\Delta \mathcal{A}^{I}\right]_{l},\left[\Delta V_{2}^{J}\right]_{s}, \Delta \mathcal{A}_{s}, \Delta \mathcal{A}_{a}\right\} ; \quad 1 \leq I \leq 6, \quad 1 \leq J \leq 2,
$$

and where the $10 \times 10$ matrix of coefficients $\mathcal{D}(\boldsymbol{\Lambda}, \omega H)$ is detailed in Appendix $\mathrm{C}$.

The critical load $\boldsymbol{\Lambda}_{c}=\left(\lambda_{1}^{c}, \widehat{h}_{c}\right)$ is found from the requirement of a nontrivial solution of the $10 \times 10$ system (3.23), i.e. from the vanishing of the determinant:

$$
\operatorname{det}\left[\mathcal{D}\left(\left(\lambda_{1}, \widehat{h}\right), \omega H\right)\right]=0 .
$$

The critical values $\boldsymbol{\Lambda}_{c}=\left(\lambda_{1}^{c}, \widehat{h}_{c}\right)$, are those roots of (3.25) that minimize $1-\lambda_{1} \geq 0$ and $\widehat{h} \geq 0$ over all non-dimensional wavenumbers $\omega H \in \mathbb{R}$, along the chosen loading path.

\section{Results and discussion}

In this section, we show the results obtained from the bifurcation analysis for the MRE substrate/layer assembly. The results are presented in three parts: in the first one, we compute the critical magnetic field as a function of the MRE substrate/layer shear moduli ratio, $G_{s} / G_{l}$ under zero applied strain along the $X_{1}$ direction, $\left(\widehat{h} \neq 0, \lambda_{1}=1\right)$. The second part addresses the effect of a purely mechanical compressive loading along the $X_{1}$ direction $\left(\widehat{h}=0, \lambda_{1}<1\right)$ as a function of the ratio $G_{s} / G_{l}$. Finally in the third part, the magnetic and mechanical loads are combined $\left(\widehat{h} \neq 0, \lambda_{1}<1\right)$. The motivation of this last part is to examine the possibility of controlling the onset of bifurcation as well the resulting wavelengths using low magnetic fields, with the ultimate goal of designing an efficient haptic device.

In the following analysis, we consider that the Lamé constant (see definitions in (2.12) and (2.14)) is for both materials $G_{s, l}^{\prime}=100 G_{s, l}$, thus exhibiting the same incompressibility for both the substrate and the MRE layer, respectively. The magnetic field is always applied in the direction of the layer thickness, i.e. $\widehat{\mathbf{h}} \| \mathbf{e}_{2}$, as shown in Fig. 1d, while the stretch is applied along the interface direction $\mathbf{e}_{1}$, as shown in Fig. 1b. The two loading configurations depicted in Fig. 1d are: the "parallel" configuration where particle chains with direction $\mathbf{N}$ are oriented parallel to the applied magnetic field $\widehat{\mathbf{h}}$ and the "perpendicular" configuration where particle chains with direction $\mathbf{N}$ are oriented perpendicular to the applied magnetic field $\widehat{\mathbf{h}}$. In the following calculations, an upper limit of the externally applied magnetic field has been set to $\widehat{h} / \rho_{0} M_{s}=4$, since at this level the magnetization $M_{2}>0.999 M_{s}$ for both the parallel and the perpendicular configuration.

\subsection{Bifurcation due to purely magnetic loading}

The graphs in Fig. 2, give in (a) the critical values for the dimensionless magnetic field $\widehat{h}_{c} / \rho_{0} M_{s}$ and in (b) the corresponding critical wavenumber $(\omega H)_{c}$ as a function of the substrate-layer shear moduli ratio $G_{s} / G_{l}$ in the absence of interface stretching $\left(\lambda_{1}=1\right)$. The applied magnetic field is always parallel to the 
a)

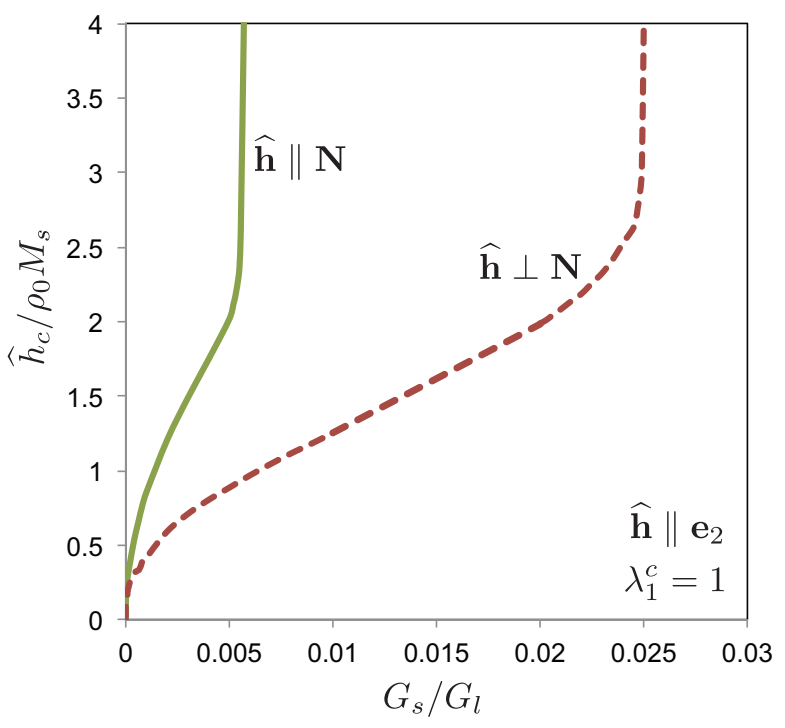

b)

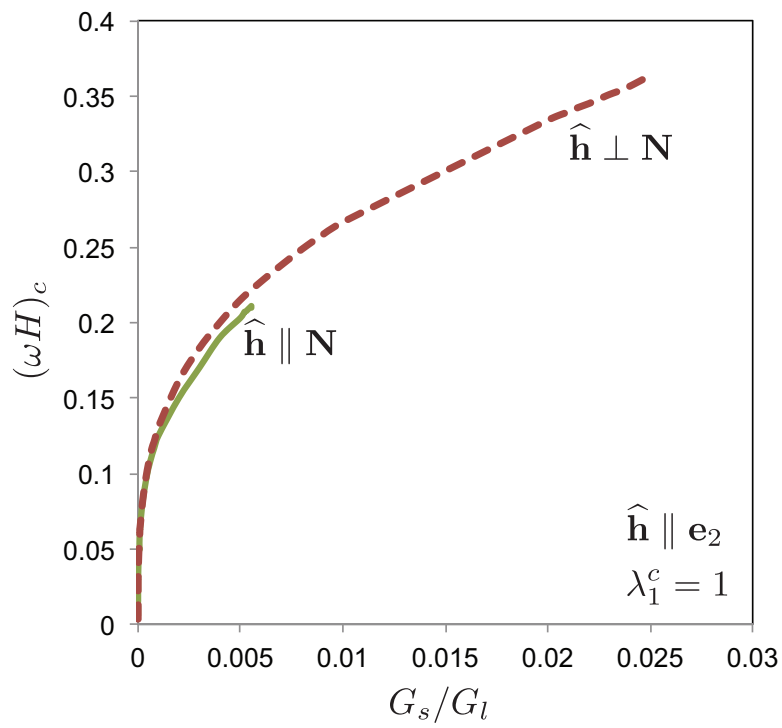

Figure 2: Critical values of the dimensionless (a) applied magnetic field $\widehat{h}_{c} / \rho_{0} M_{s}$ and (b) the corresponding wavenumber $(\omega H)_{c}$ as functions of the substrate-layer shear moduli ratio $G_{s} / G_{l}$, in the absence of interface stretching $\left(\lambda_{1}=1\right)$. The magnetic field is always parallel to the $X_{2}$ direction $\left(\widehat{\mathbf{h}} \| \mathbf{e}_{2}\right)$, while the particle chains are either parallel ( $\widehat{\mathbf{h}} \| \mathbf{N}$ - solid line) or perpendicular $(\widehat{\mathbf{h}} \perp \mathbf{N}$ - dashed line) to the applied magnetic field $\widehat{\mathbf{h}}$.

$X_{2}$ direction $\left(\widehat{\mathbf{h}} \| \mathbf{e}_{2}\right)$, while the particle chains are either parallel $(\widehat{\mathbf{h}} \| \mathbf{N}$ - solid line) or perpendicular $(\widehat{\mathbf{h}} \perp \mathbf{N}$ - dashed line) to the applied magnetic field $\widehat{\mathbf{h}}$.

As observed in Fig. 2a, a sharp asymptote is reached for the critical magnetic field $\widehat{h}_{c} / \rho_{0} M_{s}$ at finite values of $G_{s} / G_{l}$ for both cases of particle chain orientation $(\widehat{\mathbf{h}} \| \mathbf{N}$ and $\widehat{\mathbf{h}} \perp \mathbf{N})$. This is due to the fact that for an adequately high substrate stiffness, the magnetization saturation of the MRE layer ${ }^{7}$ limits the magnetic body forces, in spite of the increase of the applied magnetic field, and prevents the onset of instability. In contrast to the critical magnetic field, Fig. $2 \mathrm{~b}$ shows that the difference in the critical wavenumbers $(\omega H)_{c}$ for the parallel and the perpendicular chain orientations is negligible, at least up to $G_{s} / G_{l} \sim 0.005$ where the parallel configuration reaches magnetic saturation. However it is interesting to note in Fig. $2 \mathrm{~b}$ that no asymptote is found for the critical wavenumber $(\omega H)_{c}$ which increases monotonically with the layer-substrate shear moduli ratio, contrary to the corresponding results for the critical magnetic field $\widehat{h}_{c} / \rho_{0} M_{s}$ presented in Fig. 2a. Note that past a very small value of the substrate stiffness, the layer/substrate system does not bifurcate even at very high magnetic fields, a restricting handicap in haptic applications. A way to induce a bifurcation with low magnetic fields for a wide range of systems, that uses a combined magneto-mechanical loading, is discussed in detail in Section 4.3 .

A second remark on the results of Fig. 2 is that as $G_{s} / G_{l} \rightarrow 0$, both the critical magnetic field $\widehat{h}_{c} / \rho_{0} M_{s} \rightarrow 0$ and the corresponding critical wavenumber $(\omega H)_{c} \rightarrow 0$ (or equivalently the critical wavelength $\left.L_{c} / H \equiv 2 \pi /(\omega H)_{c} \rightarrow \infty\right)$. This result is consistent with the findings of Pao and Yeh (1973) and Kankanala and Triantafyllidis (2008) for the magnetoelastic buckling of infinitely long beams and thin blocks respectively, subjected to a transverse magnetic field, since there is no substrate deformation to oppose the onset of an infinitely long wavelength instability in the presence of an infinitesimally small magnetic field. In the presence of a substrate, the critical magnetic field $\widehat{h}_{c} / \rho_{0} M_{s}$ is a rapidly increasing function of $G_{s} / G_{l}$. Using simple structural models, we show in Appendix D that both the critical magnetic field and the critical wavenumber grow as $\widehat{h}_{c} \sim\left(G_{s} / G_{l}\right)^{1 / p}$ and $(\omega H)_{c} \sim\left(G_{s} / G_{l}\right)^{1 / p}$ (with $p>2$ ) in the neighborhood of $G_{s} / G_{l} \rightarrow 0$.

The final observation of the results in Fig. 2a pertains to the strong influence of the particle chain orientation on the critical magnetic field. Notice that for given layer and substrate stiffnesses, the critical

${ }^{7}$ Recall that the magnetization saturation in the MRE layer is described by the nonlinear term multiplying the constant $C_{6}^{*}$ in equation (2.12). For more details on the MRE constitutive response the reader is referred to Danas et al. (2012). 
magnetic field is always lower for particle chains perpendicular to the applied field $(\widehat{\mathbf{h}} \perp \mathbf{N})$ compared to the critical magnetic field corresponding to the parallel loading configuration $(\widehat{\mathbf{h}} \| \mathbf{N})$. A particle chain oriented normal to an applied magnetic field tends to rotate more easily than the one parallel to it, thus explaining why a lower magnetic field is required to destabilize the MRE layer in the first case. A related phenomenon, where particle chain orientation influences the macroscopic behavior of MRE's, is found in the recent work by Danas et al. (2012). Therein, the authors propose a micro-deformation mechanism to explain the strong influence of particle chain orientation with respect to the applied magnetic field on the experimentally observed magnetostrictive coupling in MRE's (for $\widehat{\mathbf{h}} \perp \mathbf{N}$ the magnetostrictive strains in the absence of mechanical loads are significantly higher than the corresponding strains for $\widehat{\mathbf{h}} \| \mathbf{N}$ ).

\subsection{Bifurcation due to purely mechanical loading}

As a counterpart to the study of bifurcation due to purely magnetic loads, for comparison purposes we now investigate the bifurcation of the same structure due to purely mechanical loads. This consists in revisiting the problem of a substrate/layer bifurcation subjected to axial compression, as depicted in Fig. 1b. The results recorded in Fig. 3, show the critical stretch ratio $\lambda_{1}^{c}$ in (a) and the corresponding wavenumber $(\omega H)_{c}$ in (b) as functions of the substrate/layer shear moduli ratio $G_{s} / G_{l}$. A new feature here, over the well-known classical results of the layer-on-substrate buckling problem, is the fact that the mechanical response of the layer is now defined by the transversely isotropic energy function in (2.12) (in terms of the purely mechanical invariants $I_{1}, I_{3}$ and $I_{4}$ ). This, in turn, allows us to consider two different configurations: particle chains parallel to the thickness direction ( $\mathbf{N} \| \mathbf{e}_{2}$ - solid line) and perpendicular to the thickness direction $\left(\mathbf{N} \perp \mathbf{e}_{2}-\right.$ dashed line).

a)

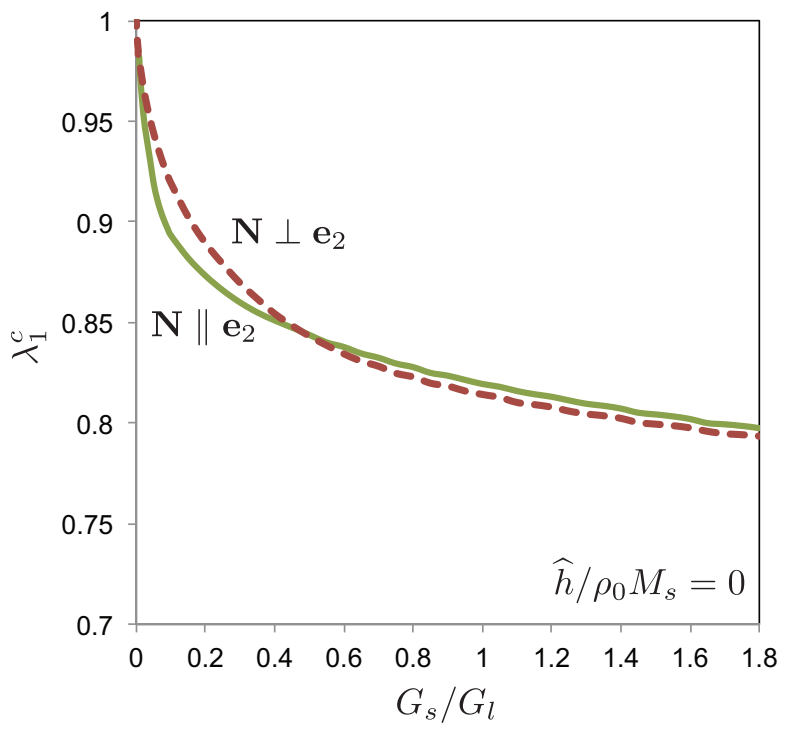

b)

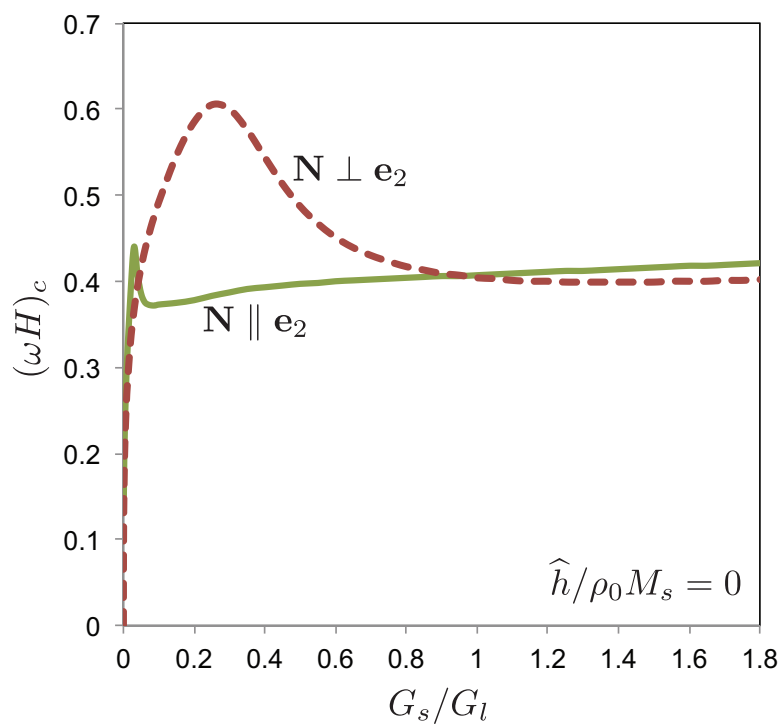

Figure 3: Critical values of (a) the interface stretch ratio $\lambda_{1}^{c}$ and (b) the corresponding wavenumber $(\omega H)_{c}$ as functions of the substrate-layer shear moduli ratio $G_{s} / G_{l}$, in the absence of external magnetic field $\left(\widehat{h} / \rho_{0} M_{s}=0\right)$. The particle chains are either parallel ( $\mathbf{N} \| \mathbf{e}_{2}$-solid line) or perpendicular ( $\mathbf{N} \perp \mathbf{e}_{2}$ - dashed line) to the thickness direction $\mathbf{e}_{2}$.

The results presented in Fig. 3a show a sharp decrease of the critical stretch ratio $\lambda_{1}^{c}<1$ as $G_{s} / G_{l}$ increases from zero (no bifurcation occurs for tensile stretch ratios $\lambda_{1}>1$, as intuitively expected). Note that the $\lambda_{1}^{c}$ vs. $G_{s} / G_{l}$ curve starts at $(1,0)$. This is due to the fact that in the absence of a substrate $G_{s} / G_{l}=0$, the critical stretch ratio is $\lambda_{1}^{c}=1$, as it corresponds to the zero compressive axial load for the Euler buckling of an infinitely long, unsupported beam. Moreover, the difference in the critical stretch ratios corresponding to the two different loading configurations ( $\mathbf{N} \| \mathbf{e}_{2}$ and $\mathbf{N} \perp \mathbf{e}_{2}$ ) is rather small and not monotonic, as observed by the crossing of the two curves. At this point it is interesting to mention that for $G_{s} / G_{l}>0.5$, the transversely isotropic mechanical response of the layer leads to lower critical strains (i.e., larger critical stretches $\lambda_{1}^{c}>0.75$ ) compared to the neo-Hookean and isotropic layer in Cao and Hutchinson (2012) not shown explicitly here. 
In Fig. 3b, the critical wavenumber $(\omega H)_{c} \rightarrow 0$ as $G_{s} / G_{l} \rightarrow 0$ in accord with the Euler solution for the axially compressed, infinitely long, unsupported beam. Away from the $G_{s} / G_{l}=0$ neighborhood, a pronounced difference between the parallel $\mathbf{N} \| \mathbf{e}_{2}$ and the $\mathbf{N} \perp \mathbf{e}_{2}$ loading cases is observed. A sharp local maximum is found in the $(\omega H)_{c}$ curve which occurs at $G_{s} / G_{l} \sim 0.03$ for the $\mathbf{N} \| \mathbf{e}_{2}$ loading and a considerably more diffuse local maximum is observed at $G_{s} / G_{l} \sim 0.3$ for the $\mathbf{N} \perp \mathbf{e}_{2}$ loading. This maximum is probably a consequence of the strongly nonlinear mechanical response of the layer even at relatively small strains, as discussed in Danas et al. (2012), while the difference between the two loading cases is due to the MRE layer's orthotropy. The critical wavenumbers approach an asymptotic limit, as they begin to become insensitive of the substrate/layer stiffness ratio for $G_{s} / G_{l}>1$. For adequately large values of $G_{s} / G_{l}$, the presence of the softer MRE layer is irrelevant, as the instability comes from the surface bifurcation of the much stiffer substrate, a phenomenon originally discovered by Biot (1965).

A word of caution is due at this point, since here we are only concerned with the onset of a bifurcation instability. In a series of papers Audoly and Boudaoud (2008a,b,c) examine the stability of bifurcated solutions and the emergence of secondary bifurcations in thin plates bonded to elastic substrates in 3D. More recently, Hutchinson (2013) has shown that the layer/substrate block can exhibit an unstable post-bifurcated regime for high enough values of $G_{s} / G_{l}$. In that work, which analyzed isotropic layers and substrates in 2D, a critical value of $G_{s} / G_{l}=0.578$ is found, beyond which the layer/substrate block could snap dynamically into creases. We emphasize that the present work pertains solely to the onset of a bifurcation in the system at hand but does not investigate the stability of the associated bifurcated path.

\subsection{Bifurcation due to combined magnetic and mechanical loading}

We finally explore the effects of a combined magneto-mechanical loading on the stability of the MRE substrate/layer assembly. In this case, the bifurcation of the structure depends on three parameters: the applied magnetic field $\widehat{h}$, the applied stretch at the interface $\lambda_{1}$ and the substrate/layer shear moduli ratio $G_{s} / G_{l}$. The influence of the particle chain orientation is also investigated.

In order to illustrate the way these three variables interact, we first fix values for the substrate/layer shear moduli ratio $G_{s} / G_{l}$. Then, we compute the critical dimensionless applied magnetic field, $\widehat{h}_{c} / \rho_{0} M_{s}$, and critical dimensionless wavenumber $(\omega H)_{c}$ as a function of the critical stretch ratio $\lambda_{1}^{c}$, as shown in Fig. 4. The particle chain orientation is, again, taken to be either parallel ( $\widehat{\mathbf{h}} \| \mathbf{N}$ - solid line) or perpendicular $(\widehat{\mathbf{h}} \perp \mathbf{N}$ - dashed line) to the applied magnetic field.

a)

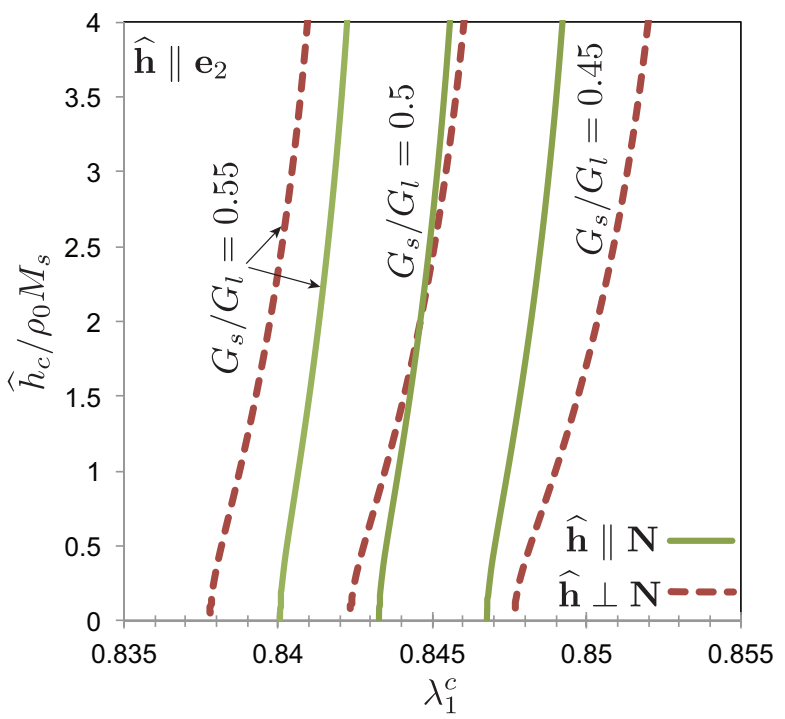

b)

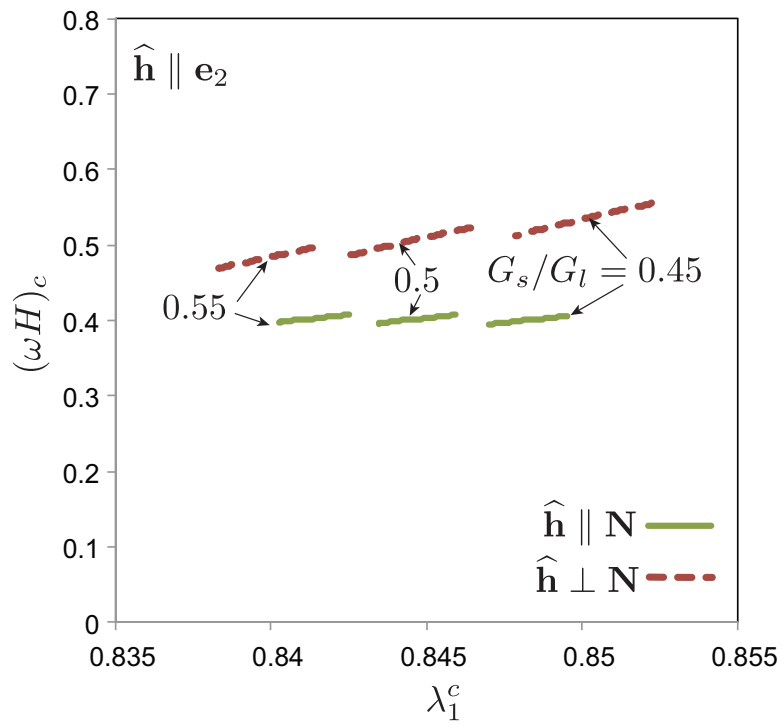

Figure 4: Critical values of the dimensionless (a) applied magnetic field $\widehat{h}_{c} / \rho_{0} M_{s}$ and (b) the corresponding wavenumber $(\omega H)_{c}$ as functions of the critical interface stretch ratio $\lambda_{1}^{c}$ for three different substrate-layer shear moduli ratios $G_{s} / G_{l}=0.45,0.5,0.55$. The magnetic field is always parallel to the $X_{2}$ direction $\left(\widehat{\mathbf{h}} \| \mathbf{e}_{2}\right)$, while the particle chains are either parallel $(\widehat{\mathbf{h}} \| \mathbf{N}$ - solid line) or perpendicular $(\widehat{\mathbf{h}} \perp \mathbf{N}-$ dashed line) to the applied magnetic field $\widehat{\mathbf{h}}$. 
In calculating the results presented in Fig. 4 , we first increase $\widehat{h}$ up to a given value and subsequently decrease $\lambda_{1}$ (away from unity) until the bifurcation conditions (3.25) are met. More specifically, in Fig. 4a, we show the dimensionless critical applied magnetic field $\widehat{h}_{c} / \rho_{0} M_{s}$ as a function of the critical interface stretch ratio $\lambda_{1}^{c}$ for three different shear moduli ratios, $G_{s} / G_{l}=0.45,0.5,0.55$, when the particle chains are parallel ( $\widehat{\mathbf{h}} \| \mathbf{N}$ - solid lines) or perpendicular $(\widehat{\mathbf{h}} \perp \mathbf{N}$ - dashed lines) to the magnetic field. As expected for $\widehat{h}=0$, each curve starts at the critical stretch ratio for the purely mechanical loading for the corresponding $G_{s} / G_{l}$, given in Fig. 3a.

The practical importance of this information lies in the fact that one could drive the substrate/layer system near (but not exactly at) a purely mechanical instability by applying an interface stretch just shy of what is needed to destabilize the system at hand and then trigger the bifurcation with a small magnetic field. Notice further that due to the crossing observed between the bifurcation curves corresponding to the parallel and perpendicular loading configurations in the purely mechanical response (see Fig. 3a), we also find a crossing between the bifurcation curves corresponding to these two configurations in Fig. 4a for $G_{s} / G_{l}=0.5$. The critical magnetic field $\widehat{h}_{c} / \rho_{0} M_{s}$ sharply increases as a function of $\lambda_{1}^{c}$ attaining an asymptote, as expected from magnetization saturation, according to the results in Fig. 2a. We can thus establish that for a given system (e.g. $G_{s} / G_{l}=0.5$ ) there is only a small range of interface stretches $\left(0.842<\lambda_{1}^{c}<0.845\right)$ in which one can obtain a bifurcation instability through an applied magnetic field.

The dimensionless critical wavenumber $(\omega H)_{c}$ results presented in Fig. $4 \mathrm{~b}$ show only weak dependence on the critical stretch $\lambda_{1}^{c}$ and the substrate/layer shear moduli ratio $G_{s} / G_{l}$. In the same figure we also observe that for the $G_{s} / G_{l}$ ratios considered in this figure, $(\omega H)_{c}$ is weakly dependent on the particle chain orientation, with the wavenumbers corresponding to the parallel loading configuration $(\widehat{\mathbf{h}} \| \mathbf{N})$ being always lower than those corresponding to the perpendicular loading configuration $(\widehat{\mathbf{h}} \perp \mathbf{N})$.

We subsequently fix values for the interface stretch ratio $\lambda_{1}^{c}<1$ and compute the critical dimensionless applied magnetic field, $\widehat{h}_{c} / \rho_{0} M_{s}$, and critical dimensionless wavenumber $(\omega H)_{c}$ as a function of the substrate/layer shear moduli ratio $G_{s} / G_{l}$. For these calculations, the particle chain orientation is either parallel $(\widehat{\mathbf{h}} \| \mathbf{N})$, for the results presented in Fig. 5 or perpendicular $(\widehat{\mathbf{h}} \perp \mathbf{N})$ to the applied magnetic field, for the results presented in Fig. 6.

a)

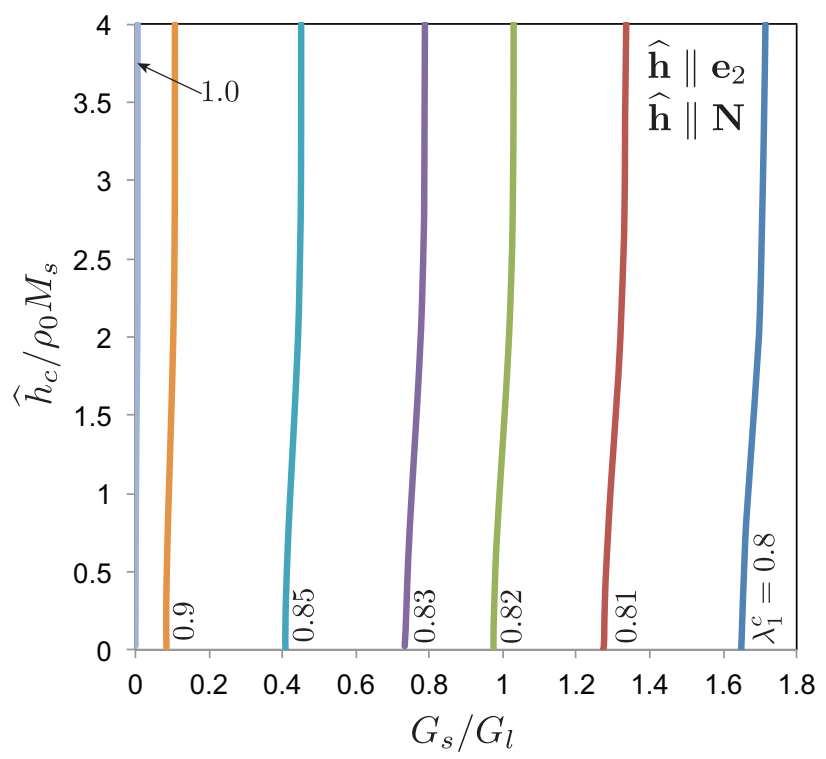

b)

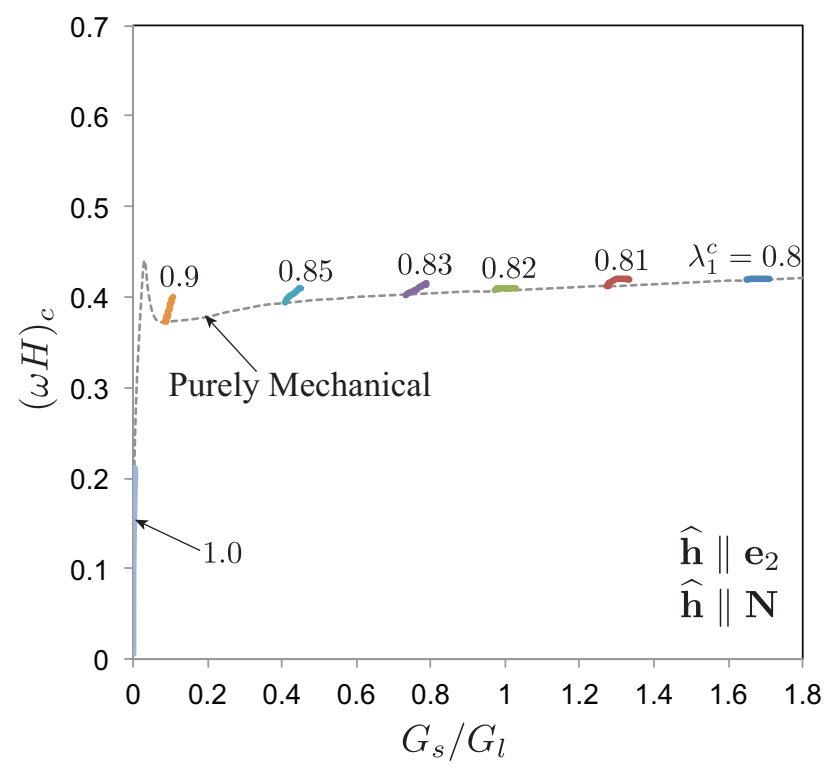

Figure 5: Critical values of the dimensionless (a) applied magnetic field $\widehat{h}_{c} / \rho_{0} M_{s}$ and (b) the corresponding wavenumber $(\omega H)_{c}$ as functions of the substrate-layer shear moduli ratio $G_{s} / G_{l}$ for seven different values of the interface stretch ratio $\lambda_{1}^{c}=0.8,0.81,0.82,0.83,0.85,0.9,1.0$. The particle chains are parallel to the magnetic field, which is always applied along $X_{2}\left(\mathbf{N}\|\widehat{\mathbf{h}}\| \mathbf{e}_{2}\right)$. The purely mechanical loading results are depicted by a dotted line (see Fig. 3b).

As expected from the results of Fig. 4 , the critical dimensionless magnetic field $\widehat{h}_{c} / \rho_{0} M_{s}$ is strongly dependent upon $\lambda_{1}$ according to the results depicted in Fig. 5a for seven different values of the interface 
stretch ratio: $\lambda_{1}^{c}=0.8,0.81,0.82,0.83,0.85,0.9,1.0$. The critical magnetic field $\widehat{h}_{c} / \rho_{0} M_{s}$ sharply increases as a function of $\lambda_{1}^{c}$ attaining an asymptote, as expected from magnetization saturation, and similarly to the results in Fig. 2a and Fig. 4a.

The practical implication of these curves is similar to the one presented for Fig. 4. For a given applied stretch (e.g., $\lambda_{1}=0.85$ ), there exist two very close extremal values of the substrate/layer shear moduli ratio $\left(0.4<G_{s} / G_{l}<0.42\right)$ for which a bifurcation can be induced through an applied magnetic field. This rather interesting behavior observed under combined compressive mechanical stretches and magnetic fields covers a very large range of values $G_{s} / G_{l}$ even when $G_{s} / G_{l}>1$, i.e. when the substrate is stiffer than the MRE layer.

The results plotted Fig. 5b show the dimensionless wavenumber $(\omega H)_{c}$ as a function of $G_{s} / G_{l}$. An asymptotic behavior for $(\omega H)_{c}$ is observed as $\lambda_{1}$ decreases and $G_{s} / G_{l}$ increases, i.e., almost the same wavenumber $(\omega H)_{c} \sim 0.4$ is obtained for a combination $\left\{\lambda_{1}=0.83, G_{s} / G_{l} \sim 0.75\right\}$ and $\left\{\lambda_{1}=0.81\right.$, $\left.G_{s} / G_{l} \sim 1.3\right\}$. The dotted line shown in the same figure corresponds to the critical wavenumber $(\omega H)_{c}$ associated with the purely mechanical instability. This, in turn, suggests that the wavelength of the critical mode is primarily set by the purely mechanical response of the system, since it changes only slightly with the application of the magnetic field, at least for finite enough stretches $\left(\lambda_{1}^{c}<0.85\right)$.

a)

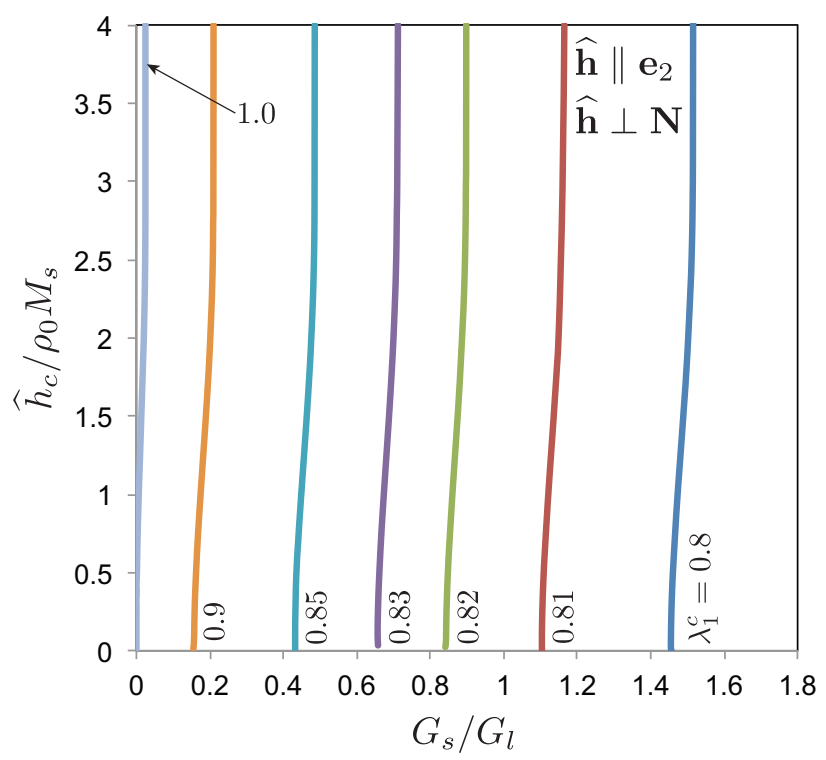

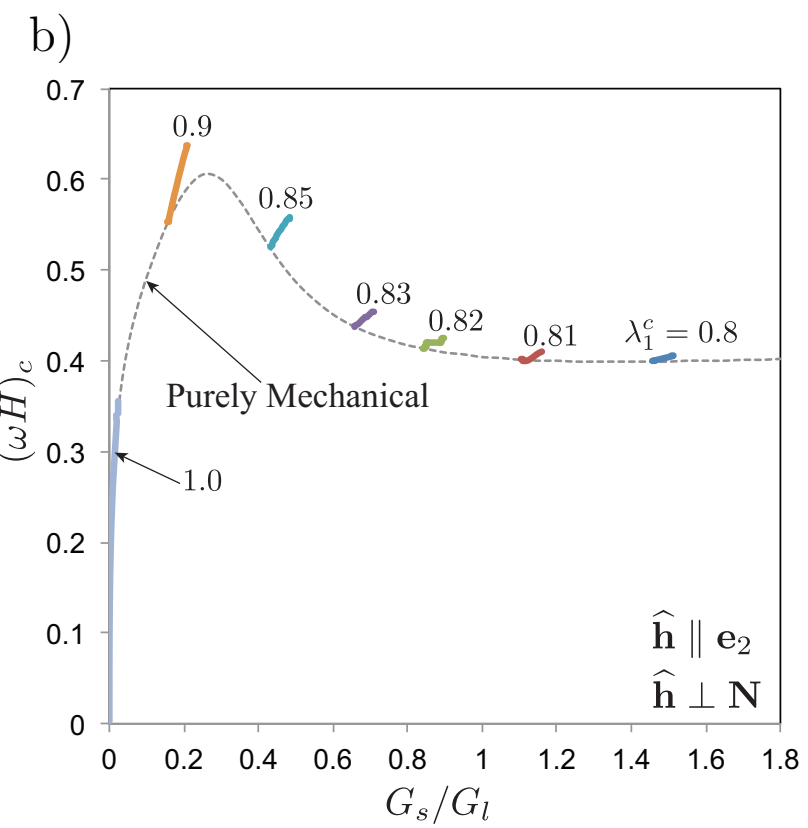

Figure 6: Critical values of the dimensionless (a) applied magnetic field $\widehat{h}_{c} / \rho_{0} M_{s}$ and (b) the corresponding wavenumber $(\omega H)_{c}$ as functions of the substrate-layer shear moduli ratio $G_{s} / G_{l}$ for seven different values of the interface stretch ratio $\lambda_{1}^{c}=0.8,0.81,0.82,0.83,0.85,0.9,1.0$. The particle chains are perpendicular to the magnetic field, which is always applied along $X_{2}\left(\mathbf{N} \perp \widehat{\mathbf{h}} \| \mathbf{e}_{2}\right)$. The purely mechanical loading results are depicted by a dotted line (see Fig. 3b).

Finally, in Fig. 6, a similar trend to the results in Fig. 5 is obtained for the case of particle chains being perpendicular to the applied magnetic field $(\widehat{\mathbf{h}} \perp \mathbf{N})$. In Fig. 6a, similar to Fig. 5 results are obtained that indicate a very small range of possible substrate/layer shear moduli ratios that can be destabilized by a magnetic field for a system with a specified $\lambda_{1}$. Interestingly, in Fig. $6 \mathrm{~b}$, the critical wavelength $(\omega H)_{c}$ reaches higher values than those in the parallel case while it reaches an asymptote for values $\lambda<0.81$ about $G_{s} / G_{l}>1.15$. The dotted line shown in this figure corresponds to the critical wavenumber $(\omega H)_{c}$ associated with the purely mechanical instability.

\section{Conclusion}

The present investigation is motivated by possible applications in haptic devices, where a transverse magnetic field can produce desirable deformation patterns in an MRE layer. The model proposed here pertains to the onset of a bifurcation in a system consisting of a transversely isotropic MRE layer 
perfectly bonded to an isotropic hyperelastic substrate and subjected to a magnetic field normal to the interface plus compressive stresses parallel to it. A 2D (plane strain) continuum model for the infinitely long substrate/layer system is used for the calculations, based on a coupled magneto-mechanical variational formulation introduced by Kankanala and Triantafyllidis (2004) and subsequently used by Kankanala and Triantafyllidis (2008) to study the magnetically-induced buckling of a rectangular block.

Under solely magnetic loading, it is found that the critical (onset-of-bifurcation) value for the applied magnetic field increases sharply with the substrate/layer shear moduli ratio. Magnetic saturation limits the possibility of such a bifurcation to very low stiffness substrates, i.e., $G_{s} / G_{l}<0.005$ and $G_{s} / G_{l}<0.025$ for the cases of particle chains oriented parallel and perpendicular to the applied magnetic field, respectively. Given the already small stiffness of the MRE layer required to maximize the magnetostrictive effect, purely magnetic loading leads to unrealistically soft substrates for haptic interface applications. To overcome this difficulty, we are proposing a combined magneto-mechanical loading that brings the system near a mechanical instability threshold by applying a lateral compression, so that a small magnetic field can subsequently trigger the desired bifurcation. A wide range of $G_{s} / G_{l}$ (even allowing for stiffer substrates $G_{s} / G_{l}>1$ ) can thus be covered by applying an adequate compression (i.e., a lateral stretch $\lambda_{1}<1$ ). It is also found that the critical wavelength is very sensitive to the microstructure: a configuration where the particle chains are perpendicular to the magnetic field (and hence parallel to the interface) allows for a much wider range of critical wavelengths than the parallel loading configuration for reasonable values of the stiffness ratio (i.e. $G_{s} / G_{l} \approx 0.3$ ). In this regard, the results presented in the previous section for the combined mechanical/magnetic loading suggest a method for designing devices that can be destabilized by using weak magnetic fields, while tuning the wavelength of the corresponding eigenmode.

Extending this analysis to a 3D setting is highly desirable for the haptic applications of interest, and is part of the planned continuation of this investigation. The present analysis pertains solely conditions for the onset of a first bifurcation. However, the response of the system is expected to be even more complex in the post-bifurcation regime, exhibiting folds and wrinkles (e.g., see the recent theoretical work on the creasing of axially compressed elastic halfspaces by Audoly and Boudaoud (2008b,c) and Zang et al. (2012) as well as the recent theoretical/experimental study on the folding wrinkles of a thin stiff layer on a soft subspace by Sun et al. (2012)), thus motivating an additional investigation direction for haptic applications. Research in these directions can proceed using the continuum anisotropic models proposed recently by Danas et al. (2012). However, the tools of homogenization theory and micro models are required in order to better explain the strong influence of the MRE's microstructure on the macroscopic stability of the system found in this work, which is another research path along which this work is planned to continue (see for instance Ponte Castañeda and Galipeau (2011) and Lopez-Pamies (2013)).

\section{Acknowledgements}

The authors would like to thank Prof. L. Bodelot of the Mechanics Department at the Ecole Polytechnique for helpful discussions.

\section{References}

Audoly, B., Boudaoud, A., 2008a. Buckling of a stiff film bound to a compliant substrateâ"part i:: Formulation, linear stability of cylindrical patterns, secondary bifurcations. Journal of the Mechanics and Physics of Solids $56,2401-2421$.

Audoly, B., Boudaoud, A., 2008b. Buckling of a stiff film bound to a compliant substrateâ"part ii:: A global scenario for the formation of herringbone pattern. Journal of the Mechanics and Physics of Solids 56, $2422-2443$.

Audoly, B., Boudaoud, A., 2008c. Buckling of a stiff film bound to a compliant substrateâ" part iii:: Herringbone solutions at large buckling parameter. Journal of the Mechanics and Physics of Solids 56, $2444-2458$.

Biot, M.A., 1937. Bending of an infinite beam on an elastic foundation. J. Appl. Mechanics A59, 1-7.

Biot, M.A., 1965. Mechanics of Incremental Deformation. Wiley, New York.

Cao, Y., Hutchinson, J.W., 2012. From wrinkles to creases in elastomers: the instability and imperfection-sensitivity of wrinkling. Proceedings of the Royal Society A: Mathematical, Physical and Engineering Science 468, 94-115.

Chen, X., Hutchinson, J.W., 2004. Herringbone buckling patterns of compressed thin films on compliant substrate. Journal of Applied Mechanics 71, 597-603.

Danas, K., Kankanala, S., Triantafyllidis, N., 2012. Experiments and modeling of iron-particle-filled magnetorheological elastomers. Journal of the Mechanics and Physics of Solids 60, $120-138$.

Ginder, J., Nichols, M., Elie, L., Tardiff, J., 1999. Magnetorheological elastomers: properties and applications. Smart Structures and Materials 1999: Smart Materials Technologies Ed. by M. Wuttig, Proc. of SPIE. 3675, $131-138$.

Huang, Z., Hong, W., Suo, Z., 2005. Nonlinear analyses of wrinkles in a film bonded to a compliant substrate. Journal of the Mechanics and Physics of Solids 53, $2101-2118$. 
Hutchinson, J.W., 2013. The role of nonlinear substrate elasticity in the wrinkling of thin films. Philosophical Transactions of the Royal Society A: Mathematical, Physical and Engineering Sciences 371. http://rsta.royalsocietypublishing.org/content/371/1993/20120422.full.pdf+html.

Kankanala, S., Triantafyllidis, N., 2008. Magnetoelastic buckling of a rectangular block in plane strain. Journal of the Mechanics and Physics of Solids 56, $1147-1169$.

Kankanala, S.V., Triantafyllidis, N., 2004. On finitely strained magnetorheological elastomers. J. Mech. Phys. Solids 52, $2869-2908$.

Lopez-Pamies, O., 2013. Elastic dielectric composites: Theory and application to particle-filled ideal dielectrics. J. Mech. Phys. Solids, in press.

Miya, K., Hara, K., Someya, K., 1978. Experimental and theoretical study on magnetoelastic buckling of a ferromagnetic cantilevered beam-plate. Journal of Applied Mechanics 45, 355-360.

Moon, F.C., Pao, Y.H., 1968. Magnetoelastic buckling of a thin plate. Journal of Applied Mechanics 35, 53-58.

Pao, Y.H., Yeh, C.S., 1973. A linear theory for soft ferromagnetic elastic solids. Int. J. Eng. Sci. $11,415-436$.

Ponte Castañeda, P., Galipeau, E., 2011. Homogenization-based constitutive models for magnetorheological elastomers at finite strain. J. Mech. Phys. Solids 59, $194-215$.

Popelar, C.H., 1972. Postbuckling analysis of a magnetoelastic beam. Journal of Applied Mechanics 39, $207-211$.

Rigbi, Z., Jilkén, L., 1983. The response of an elastomer filled with soft ferrite to mechanical and magnetic influences. J. Magn. Magn. Mater. 37, $267-276$

Rudykh, S., Bertoldi, K., 2013. Stability of anisotropic magnetorheological elastomers in finite deformations: A micromechanical approach. Journal of the Mechanics and Physics of Solids 61, $949-967$.

Shield, T.W., Kim, K.S., Shield, R.T., 1994. The buckling of an elastic layer bonded to an elastic substrate in plane strain. Journal of Applied Mechanics 61, 231-235.

Sun, J.Y., Xia, S., Moon, M.W., Oh, K.H., Kim, K.S., 2012. Folding wrinkles of a thin stiff layer on a soft substrate. Proceedings of the Royal Society A: Mathematical, Physical and Engineering Science 468, $932-953$.

Wallerstein, D.V., Peach, M.O., 1972. Magnetoelastic buckling of beams and thin plates of magnetically soft material. Journal of Applied Mechanics 39, 451-455.

Zang, J., Zhao, X., Cao, Y., Hutchinson, J.W., 2012. Localized ridge wrinkling of stiff films on compliant substrates. Journal of the Mechanics and Physics of Solids 60, 1265-1279. 


\section{A. Bifurcation equations coefficients}

The coefficients $\mathcal{L} \cdot \cdots$, required for the calculation of the second Frechet derivative of the syste's potential energy and introduced in (3.2), are given below. We remind the reader that these coefficients are calculated on the principal solution where the strains are constant and the magnetic field perturbation potential $\alpha=0$, as described in subsection 2.4.

\section{A.1. MRE layer}

For the MRE layer, the corresponding $\mathcal{L}_{\ldots . .}$ coefficients are:

$$
\begin{aligned}
& \mathcal{L}_{i j k l}^{u u}=\rho_{0}\left[\frac{\partial^{2} \psi_{l}}{\partial F_{i j} F_{k l}}+\frac{\mu_{0} \rho_{0}}{2 J}\left(M_{n} M_{n}\right)\left(F_{j i}^{-1} F_{l k}^{-1}+F_{j k}^{-1} F_{l i}^{-1}\right)\right]=\mathcal{L}_{k l i j}^{u u}, \\
& \mathcal{L}_{i j k}^{u M}=\rho_{0}\left[\frac{\partial^{2} \psi_{l}}{\partial F_{i j} M_{k}}-\frac{\mu_{0} \rho_{0}}{J} F_{j i}^{-1} M_{k}\right]=\mathcal{L}_{k i j}^{M u}, \\
& \mathcal{L}_{i j k}^{u \alpha}=\frac{\rho_{0}}{J} M_{p}\left(F_{p q} F_{j i}^{-1}-\delta_{i p} \delta_{j q}\right) \varepsilon_{q k}=\mathcal{L}_{k i j}^{\alpha u}, \\
& \mathcal{L}_{i j}^{M M}=\rho_{0}\left[\frac{\partial^{2} \psi_{l}}{\partial M_{i} M_{j}}+\frac{\mu_{0} \rho_{0}}{J} \delta_{i j}\right]=\mathcal{L}_{j i}^{M M}, \\
& \mathcal{L}_{i j}^{M \alpha}=-\frac{\rho_{0}}{J} F_{i k} \varepsilon_{k j}=\mathcal{L}_{j i}^{\alpha M}, \\
& \mathcal{L}_{i j}^{\alpha \alpha}=\frac{1}{\mu_{0} J} \varepsilon_{k i} \varepsilon_{l j} C_{k l}=\mathcal{L}_{j i}^{\alpha \alpha},
\end{aligned}
$$

where $\varepsilon_{i j}$ is the alternating symbol and $\delta_{i j}$ is the Krönecker delta. The above expressions involve derivatives of the free energy $\psi_{l}$ with respect to $\mathbf{F}$ and $\mathbf{M}$, which are calculated in terms of its invariants in Section B of the Appendix.

\section{A.2. Substrate}

For the substrate, the corresponding $\mathcal{L} \cdot \ldots$ coefficients are:

$$
\begin{aligned}
& \mathcal{L}_{i j k l}^{u u}=\rho_{0} \frac{\partial^{2} \psi_{s}}{\partial F_{i j} F_{k l}}=\mathcal{L}_{k l i j}^{u u}, \\
& \mathcal{L}_{i j}^{\alpha \alpha}=\frac{1}{\mu_{0} J} \varepsilon_{k i} \varepsilon_{l j} C_{k l}=\mathcal{L}_{j i}^{\alpha \alpha},
\end{aligned}
$$

The above expressions involve derivatives of the free energy $\psi_{s}$ with respect to $\mathbf{F}$, which are calculated in terms of its invariants in section B of the Appendix.

\section{A.3. Air}

For the air, the corresponding $\mathcal{L} \ldots$. coefficients are:

$$
\mathcal{L}_{i j}^{\alpha \alpha}=\frac{1}{\mu_{0} J} \varepsilon_{k i} \varepsilon_{l j} C_{k l}=\mathcal{L}_{j i}^{\alpha \alpha},
$$

The obvious question here is what one takes for the deformation gradient $\mathbf{F}$ in the air. From strain compatibility at the interface $F_{11}=\lambda_{1}$. The value for $F_{22}=\lambda_{2}$ can be chosen arbitrarily and is here for simplicity taken to be $\lambda_{2}=\lambda_{1}$, thus leading, according to (3.20), to $\theta_{a}=1$. A more careful examination of the boundary condition $(3.22)_{3}$, which is the only equation connecting $\mathcal{L}_{i j}^{\alpha \alpha}$ with the full eigenvalue problem, involves the combination $\mathcal{L}_{22}^{\alpha \alpha} \theta_{a}=\left(\mathcal{L}_{11}^{\alpha \alpha} \mathcal{L}_{22}^{\alpha \alpha}\right)^{1 / 2}=\left(C_{11} C_{22}\right)^{1 / 2} / J=1$, and hence independent of the choice of $\mathbf{F}$ in the air. 


\section{B. First and second derivatives of the energy density functions}

The energy density derivatives appearing in the expressions for the coefficients $\mathcal{L} . .$. in Section A of the Appendix are hereby calculated in function of the invariants of these energy densities using the standard chain rule as follows:

$$
\begin{gathered}
\frac{\partial \psi}{\partial F_{i j}}=\sum_{P=1}^{n_{i n v}} \frac{\partial \psi}{\partial I_{P}} \frac{\partial I_{P}}{\partial F_{i j}} \\
\frac{\partial \psi}{\partial M_{i}}=\sum_{P=1}^{n_{i n v}} \frac{\partial \psi}{\partial I_{P}} \frac{\partial I_{P}}{\partial M_{i}},
\end{gathered}
$$

where $n_{i n v}$ is the number of invariants relevant in each case $\left(n_{\text {inv }}=8\right.$ for the layer and $n_{\text {inv }}=2$ for the substrate). The second derivatives are similarly found to be:

$$
\begin{gathered}
\frac{\partial^{2} \psi}{\partial M_{i} \partial M_{j}}=\sum_{P=1}^{n_{i n v}} \sum_{Q=1}^{n_{i n v}} \frac{\partial^{2} \psi}{\partial I_{P} \partial I_{Q}} \frac{\partial I_{P}}{\partial M_{i}} \frac{\partial I_{Q}}{\partial M_{j}}+\sum_{P=1}^{n_{i n v}} \frac{\partial \psi}{\partial I_{P}} \frac{\partial^{2} I_{P}}{\partial M_{i} \partial M_{j}}, \\
\frac{\partial^{2} \psi}{\partial F_{i j} \partial M_{k}}=\sum_{P=1}^{n_{i n v}} \sum_{Q=1}^{n_{i n v}} \frac{\partial^{2} \psi}{\partial I_{P} \partial I_{Q}} \frac{\partial I_{P}}{\partial F_{i j}} \frac{\partial I_{Q}}{\partial M_{k}}+\sum_{P=1}^{n_{i n v}} \frac{\partial \psi}{\partial I_{P}} \frac{\partial^{2} I_{P}}{\partial F_{i j} \partial M_{k}} \\
\frac{\partial^{2} \psi}{\partial F_{i j} \partial F_{k l}}=\sum_{P=1}^{n_{i n v}} \sum_{Q=1}^{n_{i n v}} \frac{\partial^{2} \psi}{\partial I_{P} \partial I_{Q}} \frac{\partial I_{P}}{\partial F_{i j}} \frac{\partial I_{Q}}{\partial F_{k l}}+\sum_{P=1}^{n_{i n v}} \frac{\partial \psi}{\partial I_{P}} \frac{\partial^{2} I_{P}}{\partial F_{i j} \partial F_{k l}}
\end{gathered}
$$

\section{Coefficients of the matrix $\mathcal{D}$}

The coefficients of the matrix $\mathcal{D}$ defined in equation (3.23) are:

$$
\begin{aligned}
& \mathcal{D}_{1 q}=\left\{L_{1212}^{u u} \Delta \mathcal{U}_{1}^{I} \zeta_{I}+i L_{1221}^{u u} \Delta \mathcal{U}_{2}^{I}+L_{122}^{u \alpha} \zeta_{I}\right\}_{l}, \quad I=1, \ldots, 6, \quad q=I, \\
& \mathcal{D}_{1 q}=-\left\{\mathcal{L}_{1212}^{u u} \Delta \mathcal{V}_{1}^{I} \xi_{I}+i \mathcal{L}_{1221}^{u u}\right\}_{s}, \quad I=1,2, \quad q=I+6, \\
& \mathcal{D}_{2 q}=\left\{i L_{2211}^{u u} \Delta \mathcal{U}_{1}^{I}+L_{2222}^{u u} \Delta \mathcal{U}_{2}^{I} \zeta_{I}+i L_{221}^{u \alpha}\right\}_{l}, \quad I=1, \ldots, 6, \quad q=I, \\
& \mathcal{D}_{2 q}=-\left\{i \mathcal{L}_{2211}^{u u} \Delta \mathcal{V}_{1}^{I}+\mathcal{L}_{2222}^{u u} \xi_{I}\right\}_{s}, \quad I=1,2, \quad q=I+6, \\
& \mathcal{D}_{3 q}=\left\{L_{212}^{\alpha u} \Delta \mathcal{U}_{1}^{I} \zeta_{I}+i L_{221}^{\alpha u} \Delta \mathcal{U}_{2}^{I}+L_{22}^{\alpha \alpha} \zeta_{I}\right\}_{l}, \quad I=1, \ldots, 6, \quad q=I, \\
& \mathcal{D}_{39}=-\left\{\mathcal{L}_{22}^{\alpha \alpha} \theta_{s}\right\}_{s}, \quad \mathcal{D}_{3 q}=0, \quad q=7,8,10, \\
& \mathcal{D}_{4 q}=\left\{\Delta \mathcal{U}_{1}^{I}\right\}_{f}, \quad I=1, \ldots, 6, \quad q=I, \\
& \mathcal{D}_{4 q}=1, \quad q=7,8 \\
& \mathcal{D}_{5 q}=\left\{\Delta \mathcal{U}_{2}^{I}\right\}_{f}, \quad I=1, \ldots, 6, \quad q=I, \\
& \mathcal{D}_{5 q}=\left\{\Delta \mathcal{V}_{2}^{I}\right\}_{s}, \quad I=1,2, \quad q=I+6, \\
& \mathcal{D}_{6 q}=1, \quad q=1, \ldots, 6, \\
& \mathcal{D}_{69}=1, \quad \mathcal{D}_{6 q}=0, \quad q=7,8,10, \\
& \mathcal{D}_{7 q}=\left\{\left[L_{1212}^{u u} \Delta \mathcal{U}_{1}^{I} \zeta_{I}+i L_{1221}^{u u} \Delta \mathcal{U}_{2}^{I}+L_{122}^{u \alpha} \zeta_{I}\right] \exp \left(\zeta_{I} \omega H\right)\right\}_{f}, \quad I=1, \ldots, 6, \quad q=I, \\
& \mathcal{D}_{7 q}=0, q=7, \ldots, 10 \text {, } \\
& \mathcal{D}_{8 q}=\left\{\left[i L_{2211}^{u u} \Delta \mathcal{U}_{1}^{I}+L_{2222}^{u u} \Delta \mathcal{U}_{2}^{I} \zeta_{I}+i L_{221}^{u \alpha}\right] \exp \left(\zeta_{I} \omega H\right)\right\}_{f}, \quad I=1, \ldots, 6, \quad q=I, \\
& \mathcal{D}_{8 q}=0, q=7, \ldots, 10 \text {, } \\
& \mathcal{D}_{9 q}=\left\{\left[L_{212}^{\alpha u} \Delta \mathcal{U}_{1}^{I} \zeta_{I}+i L_{221}^{\alpha u} \Delta \mathcal{U}_{2}^{I}+L_{22}^{\alpha \alpha} \zeta_{I}\right] \exp \left(\zeta_{I} \omega H\right)\right\}_{f}, \quad I=1, \ldots, 6, \quad q=I, \\
& \mathcal{D}_{9 q}=0, \quad q=7,8,9, \quad \mathcal{D}_{910}=-\left\{\mathcal{L}_{22}^{\alpha \alpha} \theta_{a} \exp \left(\theta_{a} \omega H\right)\right\}_{a}, \\
& \mathcal{D}_{10 q}=\left\{\exp \left(\zeta_{I} \omega H\right)\right\}_{f}, \quad I=1, \ldots, 6, \quad q=I, \\
& \mathcal{D}_{10 q}=0, \quad q=7,8,9, \quad \mathcal{D}_{1010}=-\left\{\exp \left(\theta_{a} \omega H\right)\right\}_{a} \text {. }
\end{aligned}
$$




\section{Asymptotic result for compliant substrates}

In the limiting case where the stiffness ratio between the substrate and the MRE layer $G_{s} / G_{l} \rightarrow 0$ and the stretch $\lambda_{1}=1$, one can extract a relation between an asymptotic result for the normalized critical magnetic field $\widehat{h}_{c} / \rho_{0} M_{s}$ and the critical wavenumber $(\omega H)_{c}$ as a function of $G_{s} / G_{l}$ using "structural approximations", which lead to a scaling relation $\widehat{h}_{c} \sim(\omega H)_{c} \sim\left(G_{s} / G_{l}\right)^{1 / p}$ with $p>2$. In order to illustrate this dependence, we simplify our analysis by restricting our attention to an isotropic MRE layer and substrate, tacitly assuming that the scaling relation between $\widehat{h}_{c},(\omega H)_{c}$ and $G_{s} / G_{l}$ remains the same for the anisotropic case.

The energy density function $\psi_{l}$ of the MRE layer is approximated by:

$$
\rho_{0} \psi_{l}(\mathbf{F}, \mathbf{M})=G_{l}\left[\frac{H^{2}}{12\left(1-\nu_{l}\right)}(w, 11)^{2}+\frac{C_{7}}{2 M_{s}^{2}}\left(\lambda_{2} M\right)^{2}\right] ; w\left(X_{1}\right) \equiv u_{2}\left(X_{1}, \frac{H}{2}\right), M\left(X_{1}\right) \equiv M_{2}\left(X_{1}, \frac{H}{2}\right) .
$$

In the above expression, the mechanical part of the layer's strain energy density in (2.12) is approximated by the bending energy of the layer (Bernoulli-Euler-Navier theory) in terms of the layer's mid-plane displacement $w$, while the magnetic contribution depends only on $M$, the mid-plane, $X_{2}$-component of the specific magnetization. We only need to use the term linked to invariant $I_{7}$, since it accounts for magneto-mechanical coupling (for small strains, the contributions from the $I_{6}$ and $I_{8}$ invariants, which are also quadratic in $\mathbf{M}$, can be approximately folded in the $I_{7}$ term). The much softer substrate's energy density $\psi_{s}$ can be approximated as an elastic foundation, namely:

$$
\rho_{0} \psi_{s}=\frac{G_{s} \gamma}{2\left(1-\nu_{s}\right)} w^{2}
$$

where $\gamma$ is a constant with dimensions of (length) ${ }^{-1}$.

By using the constitutive equation for magnetization (2.9) in conjunction with the magnetic field continuity in (2.16) and the energy density approximation in (D.1):

$$
\frac{C_{7}}{M_{s}^{2}}\left(\lambda_{2}\right)^{2} M=\frac{\partial\left(\rho_{0} \psi_{l}\right)}{\partial M}=\rho_{0} \mu_{0}\left(\widehat{h}-\frac{\rho_{0}}{J}\right) M
$$

from which the following relation between $\widehat{h}$ and $M$ is found:

$$
M=\frac{\widehat{h}}{\rho_{0}\left[\beta\left(\lambda_{2}\right)^{2}+J^{-1}\right]} ; \quad \beta \equiv \frac{G_{l} C_{7}}{\mu_{0}\left(\rho_{0} M_{s}\right)^{2}} .
$$

For the case of small strains and moderate rotations approximation used here, we have the following kinematic relations for the transverse stretch ratio $\lambda_{2}$, the volume change $J=\operatorname{det} \mathbf{F}$, the strain measures $\epsilon_{11}, \epsilon_{22}$ and the slope $w, 1$ :

$$
\lambda_{2}=1+\epsilon_{22}, J=1+\epsilon_{11}+\epsilon_{22} ; \quad \epsilon_{22}=-\frac{\nu_{l}}{1-\nu_{l}} \epsilon_{11}, \epsilon_{11}=\frac{1}{2}\left(w_{, 1}\right)^{2},
$$

Substituting (D.1), (D.2), (D.4) and(D.5) into (2.1), one obtains the following approximation for the potential energy, while keeping only terms involving the displacement $w$ :

$$
\mathcal{P}=\int_{0}^{L}\left\{\frac{G_{l} H^{3}}{12\left(1-\nu_{l}\right)}\left(w_{, 11}\right)^{2}-\frac{\mu_{0} H(\widehat{h})^{2}}{4(1+\beta)^{2}} \frac{1-2 \nu_{l}+2 \beta \nu_{l}}{1-\nu_{l}}\left(w_{, 1}\right)^{2}+\frac{G_{s} \gamma}{2\left(1-\nu_{s}\right)} w^{2}\right\} \mathrm{d} X_{1} .
$$

Minimization of the above potential energy with respect to $w$, leads to the following Euler-Lagrange equation for the eigenmode $\Delta w$ :

$$
\left(\mathcal{P}_{, w w} \Delta w\right) \delta w=0 \Longrightarrow \frac{G_{l} H^{3}}{6\left(1-\nu_{l}\right)} \Delta w_{, 1111}+\frac{\mu_{0} H(\widehat{h})^{2}}{2(1+\beta)^{2}} \frac{1-2 \nu_{l}+2 \beta \nu_{l}}{1-\nu_{l}} \Delta w_{, 11}+\frac{G_{s} \gamma}{1-\nu_{s}} \Delta w=0 .
$$

By setting the eigenmode $\Delta w=W \sin \left(\omega X_{1}\right)$ in the above relation, one gets the following critical condition:

$$
\frac{G_{l}}{6\left(1-\nu_{l}\right)}(\omega H)^{4}-\frac{\mu_{0}(\widehat{h})^{2}}{2(1+\beta)^{2}} \frac{1-2 \nu_{l}+2 \beta \nu_{l}}{1-\nu_{l}}(\omega H)^{2}+\frac{G_{s} \gamma H}{1-\nu_{s}}=0 .
$$


By expressing $\widehat{h}$ as a function of $\omega H$ and minimizing over all $\omega H \in \mathbb{R}$, one obtains that the critical field $\widehat{h}_{c}$ and the corresponding dimensionless wavenumber $(\omega H)_{c}$ are:

$$
\widehat{h}_{c} \sim\left(G_{s} / G_{l}\right)^{1 / 4} ; \quad(\omega H)_{c} \sim\left(G_{s} / G_{l}\right)^{1 / 4} .
$$

Biot (1937) has proposed a modification of the substrate energy (D.2) in order to capture more accurately a sinusoidal type deformation of the substrate. This modification consists of an energy in the form $\omega w^{2} G_{s} \gamma / 2\left(1-\nu_{s}\right)$. In this case, a similar analysis to the one presented before would yield:

$$
\widehat{h}_{c} \sim\left(G_{s} / G_{l}\right)^{1 / 3} ; \quad(\omega H)_{c} \sim\left(G_{s} / G_{l}\right)^{1 / 3} .
$$

A similar conclusion has been reached by Audoly and Boudaoud (2008a) through more sophisticated analysis, who also show that this result gives a better approximation for the sinusoidal post-bifurcated equilibrium path.

In any of the two aforementioned cases, the obtained scaling relations are based on approximations of the substrate energy and they both confirm the steep increase of the critical applied magnetic field and the critical wavenumber of the numerical results in subsection 4.1. Even though, it is very difficult to judge which of the two scaling relations performs better in the present study, we find that the scaling relation corresponding to $\widehat{h}_{c} \sim\left(G_{s} / G_{l}\right)^{1 / 3}$ exhibits a larger range of validity when compared with the numerical results in Fig. 2. A more rigorous asymptotic analysis of the full field equations in (2.4), similar to the one carried out by Kankanala and Triantafyllidis (2008) is required to resolve the issue of correct scaling for the critical magnetic field for vanishingly small substrate stiffness, but it is outside the scope of this work. 